\title{
Plant use in Odo-Bulu and Demaro, Bale region, Ethiopia
}

Rainer W Bussmann ${ }^{1 *}$, Paul Swartzinsky ${ }^{2}$, Aserat Worede $^{3}$ and Paul Evangelista ${ }^{4}$

\begin{abstract}
This paper reports on the plant use of laypeople of the Oromo in Southern Ethiopia. The Oromo in Bale had names/uses for 294 species in comparison to 230 species documented in the lower reaches of the Bale area. Only 13 species was used for veterinary purposes, or as human medicine (46). Plant medicine served mostly to treat common everyday ailments such as stomach problems and diarrhea, for wound treatment and as toothbrushsticks, as anthelmintic, for skin infections and to treat sore muscles and. Interestingly, 9 species were used to treat spiritual ailments and to expel demons. In most cases of medicinal applications the leaves or roots were employed. Traditional plant knowledge has clearly declined in a large part of the research area. Western style health care services as provided by governments and NGOs, in particular in rural areas, seem to have contributed to a decline in traditional knowledge, in part because the local population simply regards western medicine as more effective and safer.
\end{abstract}

Keywords: Oromo, Ethiopia, Ethnobotany, Plant use, traditional knowledge, utilization

\section{Introduction}

Plants have been an integral part of life in many indigenous communities, and Africa is no exception [1,2]. Apart from providing building materials, fodder, weapons and other commodities, plants are especially important as traditional medicines. Many tribes and cultures in Africa have an elaborated plant knowledge-base [3]. Most of this knowledge is still entirely transferred orally within the family unit or community [4]. Western influences have, however, led to an accelerating decline of this tradition. For example, Western style healthcare supplied by some governments has been expanded in the last decades, but it is still often not readily available and many regions remain completely underserved. Subsequently, most rural communities still use herbal remedies as readily and cheaply available alternatives. This knowledge is however, rapidly dwindling due to desired changes towards a more Western lifestyle, and the influence of modern tourism and other agents of globalization.

\footnotetext{
* Correspondence: rainer.bussmann@mobot.org

${ }^{1}$ William L. Brown Center, Missouri Botanical Garden, P.O. Box 299, St. Louis, MO 63166-0299, USA

Full list of author information is available at the end of the article
}

During the last decades, a vast array of ethnobotanical studies from Ethiopia has been published. Most of these focused however on the northern regions [5-12], as well central and southern Ethiopia [13-26].

Various studies report on the toxicity and efficacy of Ethiopian traditional medicine [8,27-34].

\section{The study area}

Our study was conducted in the eastern reaches of the Bale Mountains in the southern highlands of Ethiopia (approximately $6^{\circ} 9^{\prime} \mathrm{N}, 40^{\circ} 22^{\prime} \mathrm{W}$ ) [35]. The study area covers an area approximately $380 \mathrm{~km}^{2}$ with elevations ranging from $1,500 \mathrm{~m}$ to $3,300 \mathrm{~m}$ (Figure 1). Mean minimum and maximum temperatures are $10.2 \mathrm{C}^{\circ}$ and $21.3 \mathrm{C}^{\circ}$, respectively; while mean annual precipitation ranges from 68 to $93 \mathrm{~mm}$ largely occurring during two rainy seasons. The majority of the study area is mountainous with intact forest ecosystems [36,37]. Most anthropogenic activities are centered on honey gathering and the collection of wood and bamboo (Sinarundinaria alpina). Some livestock grazing occurs, but generally at small scales. The study area has remained relatively preserved for two primary reasons: the topography is largely prohibitive to cultivation and there are two controlled hunting concessions (called Odu Bulu and
C Biomed Central

(ㄷ) 2011 BUSSMANN, RW et al; licensee BioMed Central Ltd. This is an Open Access article distributed under the terms of the Creative Commons Attribution License (http://creativecommons.org/licenses/by/2.0), which permits unrestricted use, distribution, and reproduction in any medium, provided the original work is properly cited. 


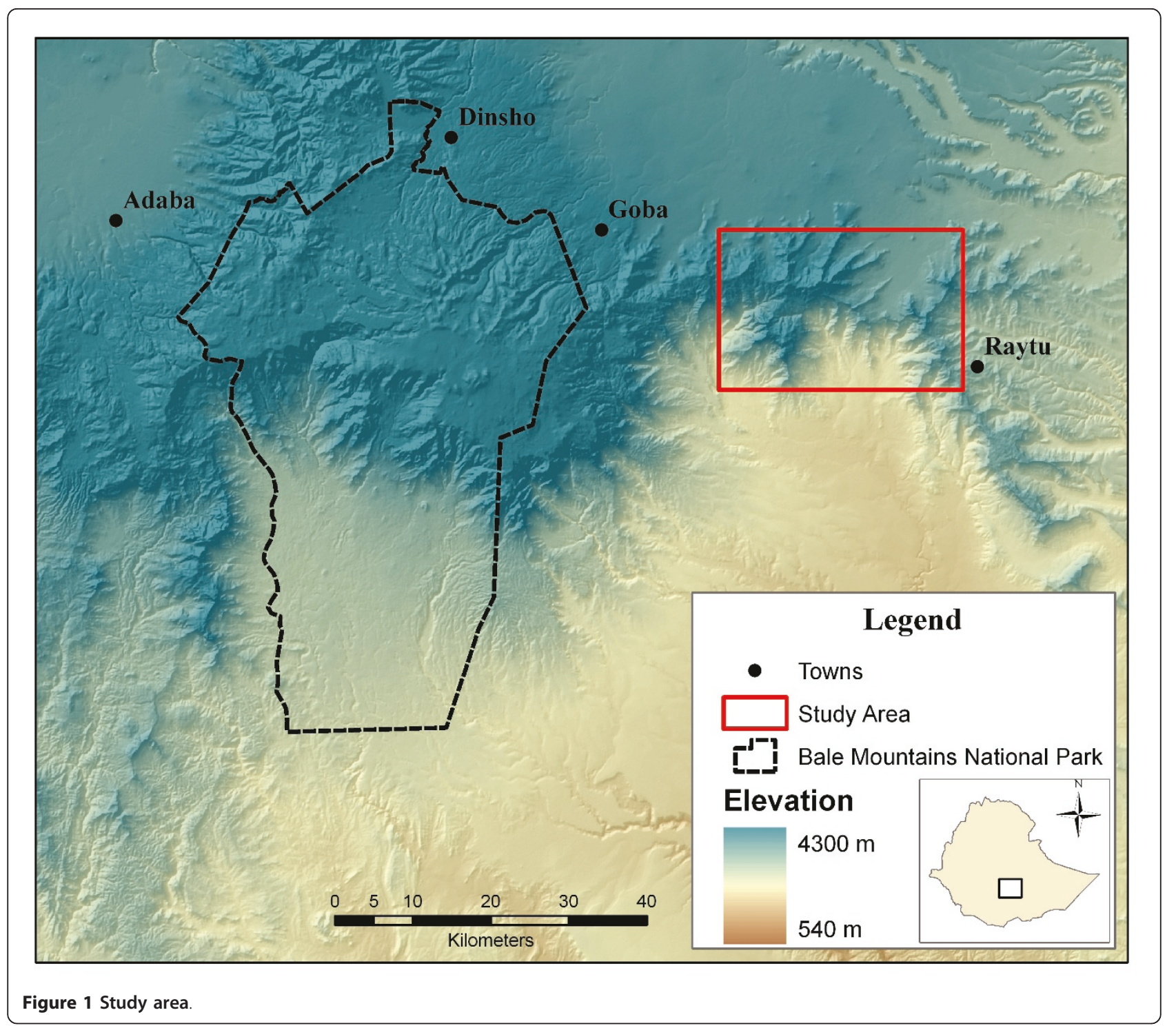

Demaro) that provide legal protection to the forest. Trophy hunting within the hunting concessions generally occurs within a three-month period; however, both concessions maintain permanent camps and guards to protect the wildlife and habitat. Just beyond the northern edges of the study area, the landscape is heavily populated with people and livestock. The forests here have long been cleared, and barley cultivation is extensive. The southern edge of the study area drops sharply in elevation before transforming into semi-arid plains that stretch into Somalia. The steep slopes act as a barrier to human and livestock encroachment providing further protection to the study area [38]. Although the study area has significantly less anthropogenic impact than nearby Bale Mountains National Park, increasing human and livestock pressure within the study area is becoming evident.

\section{The Oromo}

The Oromo are the main ethnic group in southern Ethiopia, including the Bale region, although members of many other peoples have settled in the area. Smaller populations are found in Somalia and northern Kenya. Barley and wheat cultivation provide most sustenance and income in Bale, with some areas receiving enough rainfall to support two harvests a year. Livestock keeping is also important to Oromo people, but occurs to a lesser extent than most areas in Ethiopia. During the time when crops are cultivated, livestock are grazed in the forest and Afro-alpine of higher elevations. Because some areas can support two harvests annually, livestock may spend as long as ten months in natural areas. During the last decades, Bale has seen profound changes, from increased access and governmental health care entering during the communist era of the 1970s and 
80 s, to an increase in tourism in the 1990 s and a large influx of Chinese development aid in the last few years. These years have also marked a dramatic increase in human and livestock populations, and consequently land-use and conversion of the landscape. Throughout the 1980s and early 90s, Ethiopia's communist government regularly relocated people from northern regions to Bale as a means to disrupt civil opposition [39]. Since then, the current government has continued the practice on a voluntary basis as an effort to provide people access to natural resources, which have been depleted in other parts of the country. Collectively, these events have put an enormous strain on forests in the Bale Mountains, and are changing the local economy and traditional customs profoundly.

The ethnobotany of various subgroups of the Oromo has been focal point of a few recent studies [40-42].

\section{Materials and Methods}

\section{Ethnobotanical data and plant collections}

Fieldwork was carried out between 2009 by Bussmann and collaborators. To obtain information on plants used traditionally, interviews were conducted using semistructured questionnaires [43]. Random sampling technique was applied in distributing the questionnaires. Before carrying out the interviews, an oral prior informed consent was sought from every respondent. All communities involved showed the same acceptance of the researchers, and similar in-field times were involved in the study in order to avoid possible errors in data depth.

A total of 12 lay respondents were interviewed. Access to female informants was not possible. In order to get a more detailed inventory of plant use, ethnobotanical data were collected by conducting interviews directly in the field during collection trips, and by discussing the freshly collected specimens with informants, after seeking oral consent from each respondent. This method was preferred over pure questionnaires to also get an indication for species that are not used by the community, and which are normally not mentioned during traditional interviews. All interviews were carried out in local language by native speakers, and then translated into English. Voucher specimens were collected and are preserved at the National Herbarium of Ethiopia (ADD). The identification of plant material followed the Flora of Ethiopia and Erithrea [44-50], as well as [51-53]. Plant nomenclature follows TROPICOS http://www.tropicos.org.

\section{Results and Discussion}

The Oromo in Bale had names/uses for 294 species encountered (Table 1.), in comparison to 230 species documented in the lower reaches of the Bale area [23], and 101 species in the highlands [41]. The latter study did however interview health experts, while the present work focused on the knowledge of laypeople. One hundred and sixty two species encountered in this study were classified as having no uses whatsoever, although many of them were named. Many of the identified species had multiple uses or were known provide important direct or indirect services to the community (Figure 2). Most species named (172 species) were used for livestock grazing (mostly cattle). The vernacular name "Marga" for many Poaceae simply translates to "grass", and underlines the importance of this resource. It is important to note however that 42 of these were also indicated to be important for the endemic and endangered mountain nyala (Tragelaphus buxtoni), illustrating a potential conflict between pastoralist use and wildlife conservation. A further 27 species were used as fodder for both domestic animals and eaten by wildlife. Again the vernacular names often pointed to that specific use. Argemone mexicana and similar spiny species were all called "Korehare" which translates to "spiny donkey", and all serve as fodder for donkeys. Nine species were used as poisons against carnivores. Fifty-one tree species were used as firewood, while only two served to produce charcoal. Traditional houses are to a large extent built using material from the forest, and it is not astonishing that 15 tree species were used for timber, 17 species provided material to make ropes, mostly used to tie the house posts and roof beams, and 10 species were used as thatch. A wide variety of plants was found to be employed for the fabrication of tools and household implements ( 3 for brooms, 4 to make beehives, 3 for tanning, 11 to make ploughs, 2 served as detergent to wash clothes). In addition forest species were an important source of nutrients, with 28 species collected as food, and 23 explicitly used for honey production.

A very limited number of species was used for veterinary purposes (13 species), or as human medicine (46 species). Many species however had multiple uses. Plant medicine served mostly to treat common everyday ailments such as stomach problems and diarrhea (9 species), for wound treatment and as toothbrush-sticks (6 species), as anthelmintic, for skin infections and to treat sore muscles and swellings (4 species each), or to foster hair growth, to treat colds, and syphilis (2 species each). One species was employed for female illnesses, and one to treat cancer. Interestingly, 9 species were used to treat spiritual ailments and to expel demons. In most cases of medicinal applications the leaves (26 species) or roots (15 species) were employed, while fruits (4 species), flowers (1 species) and bark (1 species) did not play a significant role.

Traditional plant knowledge has clearly declined in a large part of the research area. The most traditional groups still retain the highest knowledge of plant use for 
Table 1 Plants encountered in Odo Bulu and Demaro, Bale, Ethiopia

\begin{tabular}{|c|c|c|c|}
\hline Voucher & Oromifa & Family & Scientific name \\
\hline 16191 & Sokoro & Acanthaceae & Acanthus sp. \\
\hline 16011 & Sokoro & Acanthaceae & Acanthus eminens C.B. Clarke \\
\hline 16011 & Sokoro & Acanthaceae & Acanthus eminens C.B. Clarke \\
\hline 16011 & Sokoro & Acanthaceae & Acanthus sennii Chiov. \\
\hline 16236 & Sokoru & Acanthaceae & Acanthus sennii Chiov. \\
\hline 16223 & Dergu & Acanthaceae & Dicliptera laxata C.B. Clarke \\
\hline 16210 & Dergu & Acanthaceae & Dicliptera sp. \\
\hline 16210 & Dergu & Acanthaceae & Dicliptera sp. \\
\hline 15999 & Dergu & Acanthaceae & Hypoestes forskaolii (Vahl.) R. Br. \\
\hline 16293 & Gurbi & Acanthaceae & Hypoestes sp. \\
\hline 16220 & Dergu & Acanthaceae & $\begin{array}{l}\text { Hypoestes triflora (Forssk.) Roem. \& } \\
\text { Schult. }\end{array}$ \\
\hline 16046 & Dergu & Acanthaceae & Justicia diclipteroides Lindau \\
\hline 16263 & Gurbi & Acanthaceae & Justicia diclipteroides Lindau \\
\hline 16336 & Umuga & Acanthaceae & $\begin{array}{l}\text { Justicia schimperiana (Hochst. Ex } \\
\text { Nees) T. Anderson }\end{array}$ \\
\hline 16288 & Gurbi & Acanthaceae & Justicia sp. \\
\hline 16040 & Herraye & Acanthaceae & Minulopsis solmsii Schweinf. \\
\hline
\end{tabular}

Uses and notes

No use.

Flowers for honey.

NOT eaten by animals

Medicine; leaves are dried, ground, mixed with butter and applied to wounds.

Flowers for honey.

Eaten by cattle and mountain nyala.

NOTE: "Flower is different from place to place".

Eaten by cattle.

Flowers for honey.

Eaten by animals.

Eaten by cattle and mountain nyala.

Eaten by cattle and mountain nyala.

Eaten by cattle, bushbuck and mountain nyala.

Eaten by cattle.

Rope; bark peeled and used as rope for construction to attach the wall fragments.

Eaten by cattle and mountain nyala.

Flowers for honey.

NOTE:

"This flowers only once in seven years. I have seen it twice in my lifetime (the speaker was about 55 years old). The last

flowering was about 10 years ago when the great rain stopped. (El Niño 1998). It also flowered before the king was replaced (around 1973).

\begin{tabular}{|c|c|c|c|}
\hline 16237 & Anano & Acanthaceae & Thunbergia alata Bojer ex Sims \\
\hline 16294 & Gurbi & Acanthaceae & \\
\hline 16313 & Gurbi & Acanthaceae & \\
\hline 16397 & No name & Acanthaceae & \\
\hline 6408 & No name & Acanthaceae & \\
\hline 16228 & Hacho & Amaranthaceae & Achyranthes aspera L. \\
\hline 16228 & Hacho & Amaranthaceae & Achyranthes aspera L. \\
\hline 16144 & Rafu & Amaranthaceae & Amaranthus sp. \\
\hline & Rafu & Amaranthaceae & Amaranthus sp. \\
\hline 16153 & Ch'okene & Amaranthaceae & Amaranthus sp. \\
\hline 16153 & Ch'okene & Amaranthaceae & Amaranthus sp. \\
\hline 16153 & Ch'okene & Amaranthaceae & Amaranthus sp. \\
\hline 16355 & Rafu & Amaranthaceae & Amaranthus sp. \\
\hline 16 & Rafu & Amaranthaceae & Amaranthus sp. \\
\hline & Gurbi & Amaranthaceae & Celosia anthelminthica Aschers. \\
\hline 16007 & Hacho & Amaranthaceae & Cyathula cylindrica Moq. \\
\hline 16007 & Hacho & Amaranthaceae & Cyathula cylindrica Moq. \\
\hline 16247 & Hacho & Amaranthaceae & Cyathula polycephala Baker \\
\hline & Hacho & Amaranthaceae & $\begin{array}{l}\text { Cyathula uncinulata (Schrad.) } \\
\text { Schinz }\end{array}$ \\
\hline 16127 & Hacho & Amaranthaceae & $\begin{array}{l}\text { Cyathula uncinulata (Schrad.) } \\
\text { Schinz }\end{array}$ \\
\hline 6 & Hacho & Amaranthaceae & $\begin{array}{l}\text { Cyathula uncinulata (Schrad.) } \\
\text { Schinz }\end{array}$ \\
\hline
\end{tabular}

Eaten by cattle and mountain nyala.

Eaten by cattle and mountain nyala.

Eaten by cattle and mountain nyala.

No use.

No use.

Eaten by cattle.

Veterinary; crush the root, boil it in water and give the animals to drink against rabies.

Eaten by cattle.

Food; cooked like cabbage in time of drought.

Medicine; as remedy for spiritual pain. Inhaling the smell brings out the evil.

NOTE: Name translates to "tall".

NOT eaten by livestock.

Veterinary; crushed and smeared on cattle for spiritual protection and "highsight".

Eaten by cattle.

No use.

Eaten by cattle and mountain nyala.

Eaten by cattle.

Veterinary; crush the root, boil it in water and give the animals to drink against rabies.

Eaten by cattle.

Eaten by cattle.

Veterinary; crush the root, boil it in water and give the animals to drink against rabies.

Eaten by cattle. 
Table 1 Plants encountered in Odo Bulu and Demaro, Bale, Ethiopia (Continued)

\begin{tabular}{|c|c|c|c|c|}
\hline 16216 & Hacho & Amaranthaceae & $\begin{array}{l}\text { Cyathula uncinulata (Schrad.) } \\
\text { Schinz }\end{array}$ & $\begin{array}{l}\text { Veterinary; crush the root, boil it in water and give the animals } \\
\text { to drink against rabies. }\end{array}$ \\
\hline 16297 & No name & Amaranthaceae & & No use. \\
\hline 16376 & Komudu & Amaranthaceae & & No use. \\
\hline 16289 & Tadesa & Anacardiaceae & Rhus sp. & Eaten by cattle and mountain nyala. \\
\hline 16289 & Tadesa & Anacardiaceae & Rhus sp. & Food; children eat the sweet fruits. \\
\hline 16213 & No name & Apiaceae & $\begin{array}{l}\text { Agrocharis incognita (C. Norman) } \\
\text { Heyw. \& Jury }\end{array}$ & Eaten by cattle. \\
\hline 16213 & No name & Apiaceae & $\begin{array}{l}\text { Agrocharis incognita (C. Norman) } \\
\text { Heyw. \& Jury }\end{array}$ & Medicine; root is crushed and eaten for stomach problems. \\
\hline 16213 & No name & Apiaceae & $\begin{array}{l}\text { Agrocharis incognita (C. Norman) } \\
\text { Heyw. \& Jury }\end{array}$ & $\begin{array}{l}\text { Veterinary; root is crushed and given to livestock for stomach } \\
\text { problems. }\end{array}$ \\
\hline 15986 & No name & Apiaceae & Carum sp. & No use. \\
\hline 16182 & No name & Apiaceae & Hydrocotyle mannii Hook.f. & Eaten by baboons. \\
\hline 16010 & $\begin{array}{l}\text { Informant does } \\
\text { not remember } \\
\text { name }\end{array}$ & Apiaceae & Pimpinella oreophila Hook. f. & Eaten by livestock and wildlife. \\
\hline 16010 & $\begin{array}{l}\text { Informant does } \\
\text { not remember } \\
\text { name }\end{array}$ & Apiaceae & Pimpinella oreophila Hook. f. & $\begin{array}{l}\text { Medicine; roots are ground and prepared as tea for stomach } \\
\text { problems. }\end{array}$ \\
\hline 15987 & No name & Apiaceae & $\begin{array}{l}\text { Sanicula elata Buch.-Ham. ex D. } \\
\text { Don. }\end{array}$ & No use. \\
\hline 15993 & $\begin{array}{l}\text { Informant does } \\
\text { not remember } \\
\text { name }\end{array}$ & Apiaceae & Torilis arvensis (Huds.) Link & Eaten by animals. \\
\hline 16088 & No name & Apiaceae & & No use. \\
\hline 16115 & No name & Apiaceae & & No use. \\
\hline 16171 & No name & Apiaceae & & Eaten by baboons. \\
\hline 16326 & Hagamsa & Apocynaceae & Carissa edulis (Forssk.) Vahl & Eaten by goats. \\
\hline 16326 & Hagamsa & Apocynaceae & Carissa edulis (Forssk.) Vahl & Food; fruit eaten by humans. \\
\hline 16027 & Homba & Apocynaceae & Oncinotis tenuiloba Stapf. & $\begin{array}{l}\text { Eaten by livestock and mountain nyala. } \\
\text { NOTE: The latex is very sticky but not poisonous. }\end{array}$ \\
\hline 16027 & Homba & Apocynaceae & Oncinotis tenuiloba Stapf. & $\begin{array}{l}\text { Rope. } \\
\text { NOTE: The latex is very sticky but not poisonous. }\end{array}$ \\
\hline 16423 & Bulala & Apocynaceae & Oncinotis tenuiloba Stapf. & Rope for construction. \\
\hline 16330 & Diki & Apocynaceae & & Eaten by cattle and mountain nyala (leaves). \\
\hline 16330 & Diki & Apocynaceae & & Rope; bark peeled and used as rope for construction. \\
\hline 16333 & Gidila & Apocynaceae & & $\begin{array}{l}\text { Poison; used to kill carnivores (mainly hyenas and lions); dry } \\
\text { plant, crush and put on meat. }\end{array}$ \\
\hline 16400 & Anano & Apocynaceae & & Eaten by cattle and mountain nyala. \\
\hline 16422 & Anano & Apocynaceae & & Eaten by cattle and mountain nyala. \\
\hline 16031 & Abeye & Aquifoliaceae & Ilex mitis (L.) Radkl. & $\begin{array}{l}\text { Food; women use the leaves to roll the dough in before } \\
\text { putting it in the oven so that it does not burn. The seeds are } \\
\text { crused and the oil is used to grease the baking plate before } \\
\text { baking. }\end{array}$ \\
\hline 16414 & Arfatu & Araliaceae & Cussonia holstii Harms ex Engl. & Beehives (wood). \\
\hline 16214 & Gatami & Araliaceae & $\begin{array}{l}\text { Schefflera abyssinica } \\
\text { (Hochst. ex Rich.) Harms }\end{array}$ & Firewood. \\
\hline 16214 & Gatami & Araliaceae & $\begin{array}{l}\text { Schefflera abyssinica } \\
\text { (Hochst. ex Rich.) Harms }\end{array}$ & Flowers for honey. \\
\hline 16214 & Gatami & Araliaceae & $\begin{array}{l}\text { Schefflera abyssinica } \\
\text { (Hochst. ex Rich.) Harms }\end{array}$ & NOT eaten by cattle. \\
\hline 16025 & Ansha & Araliaceae & Schefflera volkensii (Harms) Harms & Eaten by cattle and colobus monkeys. \\
\hline 16025 & Ansha & Araliaceae & Schefflera volkensii (Harms) Harms & Firewood. \\
\hline 16041 & Ansha & Araliaceae & Schefflera volkensii (Harms) Harms & Firewood. \\
\hline 16043 & Meti & Arecaceae & Phoenix reclinata Jacq. & Weave wedding baskets and floor mats. \\
\hline 16134 & Seriti & Asparagaceae & Asparagus africanus Lam. & Eaten by cattle and wildlife. \\
\hline
\end{tabular}


Table 1 Plants encountered in Odo Bulu and Demaro, Bale, Ethiopia (Continued)

\begin{tabular}{|c|c|c|c|c|}
\hline 16134 & Seriti & Asparagaceae & Asparagus africanus Lam. & Medicine; crush the plant, extract the juice and put on pimples. \\
\hline 16337 & Sariti & Asparagaceae & Asparagus africanus Lam. & Making brooms. \\
\hline 16135 & Seriti & Asparagaceae & Asparagus falcatus L. & Eaten by cattle and wildlife. \\
\hline 16135 & Seriti & Asparagaceae & Asparagus falcatus L. & Medicine; crush the plant, extract the juice and put on pimples. \\
\hline 16202 & No name & Asparagaceae & Chlorophytum ducis-aprutii Chiov. & No use. \\
\hline 15990 & Kokosa & Aspleniaceae & Asplenium friesiorum C. Chr. & No use. \\
\hline 16017 & Kokosa & Aspleniaceae & Asplenium monanthes L. & Sometimes eaten by cattle and mountain nyala. \\
\hline 16013 & Kokosa & Aspleniaceae & Asplenium sp. & Sometimes eaten by cattle and mountain nyala. \\
\hline 16014 & Kokosa & Aspleniaceae & Asplenium sp. & Sometimes eaten by cattle and mountain nyala. \\
\hline 16099 & Kokosa & Aspleniaceae & Asplenium sp. & No use. \\
\hline 16120 & Kokosa & Aspleniaceae & Asplenium sp. & No use. \\
\hline 16196 & Kokosa & Aspleniaceae & Asplenium sp. & No use. \\
\hline 16197 & Kokosa & Aspleniaceae & Asplenium sp. & No use. \\
\hline 16198 & Kokosa & Aspleniaceae & Asplenium sp. & No use. \\
\hline 16206 & No name & Aspleniaceae & Asplenium sp. & No use. \\
\hline 16207 & Kokosa & Aspleniaceae & Asplenium sp. & No use. \\
\hline 16012 & Kokosa & Aspleniaceae & Asplenium theciferum (Kunth.) Mett. & Sometimes eaten by cattle and mountain nyala. \\
\hline 16244 & Gurbi & Asteraceae & Ageratum conyzoides L. & No use. \\
\hline 16382 & Kore'apata & Asteraceae & Arctium lappa L. & No use. \\
\hline 16147 & Ch'okone & Asteraceae & Artemisia absinthium (Mill.) DC. & $\begin{array}{l}\text { Medicine; as remedy for spiritual pain. Inhaling the smell brings } \\
\text { out the evil. } \\
\text { NOTE: Name translates to "tall". }\end{array}$ \\
\hline 16147 & Ch'okone & Asteraceae & Artemisia absinthium (Mill.) DC. & $\begin{array}{l}\text { NOT eaten by livestock. } \\
\text { NOTE: Name translates to "tall". }\end{array}$ \\
\hline 16147 & Ch'okone & Asteraceae & Artemisia absinthium (Mill.) DC. & $\begin{array}{l}\text { Veterinary; crushed and smeared on cattle for spiritual } \\
\text { protection and "highsight". } \\
\text { NOTE: Name translates to "tall". }\end{array}$ \\
\hline 16354 & $\begin{array}{l}\text { Informant does } \\
\text { not remember } \\
\text { name }\end{array}$ & Asteraceae & Artemisia absinthium (Mill.) DC. & No use. \\
\hline 16113 & Hada & Asteraceae & Bidens sp. & Eaten by cattle. \\
\hline 16190 & Korehare & Asteraceae & $\begin{array}{l}\text { Carduus nyassanus (S. Moore) R.E. } \\
\text { Fr. }\end{array}$ & $\begin{array}{l}\text { Eaten by donkeys. } \\
\text { NOTE: Name translates to "spiny donkey". }\end{array}$ \\
\hline 16125 & No name & Asteraceae & Centaurea sp. & No use. \\
\hline 16039 & No name & Asteraceae & Cineraria deltoidea Sond. & Eaten by cattle. \\
\hline 16122 & Korehare & Asteraceae & Cirsium dender Friis & $\begin{array}{l}\text { Eaten by donkeys. } \\
\text { NOTE: Name translates to "spiny donkey". }\end{array}$ \\
\hline 16368 & Korehare & Asteraceae & Cirsium vulgare (Savi) Ten. & $\begin{array}{l}\text { Eaten by donkeys. They prefer the heads. } \\
\text { NOTE: Name translates to "spiny donkey". }\end{array}$ \\
\hline 15998 & $\begin{array}{l}\text { Informant does } \\
\text { not remember } \\
\text { name }\end{array}$ & Asteraceae & Crassocephalum sp. & Eaten by cattle. \\
\hline 16038 & No name & Asteraceae & Crassocephalum sp. & Eaten by cattle. \\
\hline 16409 & No name & Asteraceae & Crepis cf. rueppellii Sch. Bip. & No use. \\
\hline 16052 & Korehare & Asteraceae & Echinops hoehnelii Schweinf. & Eaten by donkeys. \\
\hline 16204 & No name & Asteraceae & Galinsoga parviflora Cav. & No use. \\
\hline 16108 & No name & Asteraceae & Gnaphalium sp. & Eaten by cattle. \\
\hline 16145 & $\begin{array}{l}\text { Informant does } \\
\text { not remember } \\
\text { name }\end{array}$ & Asteraceae & $\begin{array}{l}\text { Haplocarpha rueppellii (Sch. Bip.) K. } \\
\text { Lewin }\end{array}$ & Eaten by cattle. \\
\hline 16176 & No name & Asteraceae & $\begin{array}{l}\text { Helichrysum formosissimum Sch. } \\
\text { Bip. }\end{array}$ & No use. \\
\hline 16093 & No name & Asteraceae & Helichrysum sp. & No use. \\
\hline 16094 & No name & Asteraceae & Helichrysum sp. & No use. \\
\hline 16142 & No name & Asteraceae & Helichrysum sp. & No use. \\
\hline 16357 & Hariti & Asteraceae & Helichrysum sp. & No use. \\
\hline
\end{tabular}


Table 1 Plants encountered in Odo Bulu and Demaro, Bale, Ethiopia (Continued)

\begin{tabular}{|c|c|c|c|c|}
\hline 16055 & Hatawi & Asteraceae & Inula confertiflora A. Rich. & $\begin{array}{l}\text { Poison; this is NOT eaten by animals. The leaves look like nice } \\
\text { toilet paper but should not be used because they will cause } \\
\text { swellings. }\end{array}$ \\
\hline 15988 & No name & Asteraceae & Mikania sp. & Eaten by cattle. \\
\hline 16002 & Karkora & Asteraceae & $\begin{array}{l}\text { Mikaniopsis clematoides Milne- } \\
\text { Redh. }\end{array}$ & Eaten by cattle and mountain nyala. \\
\hline 16205 & No name & Asteraceae & Mikaniopsis sp. & $\begin{array}{l}\text { Medicine; "eye medicine" for better spiritual view (chewed } \\
\text { root). The root is also chewed to protect against Evil Eye. }\end{array}$ \\
\hline 16161 & Anono & Asteraceae & Prenanthes subpeltata Stebbins & $\begin{array}{l}\text { Medicine; leaves are boiled and then put on swellings and } \\
\text { bruises. }\end{array}$ \\
\hline 16165 & Anono & Asteraceae & Prenanthes subpeltata Stebbins & $\begin{array}{l}\text { Medicine; leaves are boiled and then put on swellings and } \\
\text { bruises. }\end{array}$ \\
\hline 16037 & Hagedena & Asteraceae & Senecio sp. & Flowers for honey. \\
\hline 16089 & No name & Asteraceae & Senecio sp. & Eaten by cattle. \\
\hline 16095 & Adado & Asteraceae & Senecio sp. & Rope; to tie the main pole of the house. Very durable. \\
\hline 16095 & Adado & Asteraceae & Senecio sp. & Firewood. \\
\hline 16114 & No name & Asteraceae & Senecio sp. & No use. \\
\hline 16174 & No name & Asteraceae & Senecio sp. & No use. \\
\hline 16175 & No name & Asteraceae & Senecio sp. & No use. \\
\hline 16430 & Buritaro & Asteraceae & Senecio sp. & No use. \\
\hline 16131 & Rafu & Asteraceae & $\begin{array}{l}\text { Solanecio angulatus (Vahl) C. } \\
\text { Jeffrey }\end{array}$ & $\begin{array}{l}\text { Medicine; the leaves are boild and the steam inhaled for } \\
\text { spiritual cleansing and to expel spirits in crazy people. }\end{array}$ \\
\hline 16131 & Rafu & Asteraceae & $\begin{array}{l}\text { Solanecio angulatus (Vahl) C. } \\
\text { Jeffrey }\end{array}$ & NOT eaten by cattle. \\
\hline 16132 & Galesimbira & Asteraceae & Sonchus bipontini Asch. & $\begin{array}{l}\text { Eaten by cattle (given to calves to strengthen them). } \\
\text { NOTE: Name translates to "birdvine". }\end{array}$ \\
\hline 16132 & Galesimbira & Asteraceae & Sonchus bipontini Asch. & $\begin{array}{l}\text { Eaten by cattle. } \\
\text { NOTE: Name translates to "birdvine". }\end{array}$ \\
\hline 16132 & Galesimbira & Asteraceae & Sonchus bipontini Asch. & $\begin{array}{l}\text { Medicine; used for swellings. } \\
\text { NOTE: Name translates to "birdvine". }\end{array}$ \\
\hline 16132 & Galesimbira & Asteraceae & Sonchus bipontini Asch. & $\begin{array}{l}\text { Veterinary; used for swellings. } \\
\text { NOTE: Name translates to "birdvine". }\end{array}$ \\
\hline 16166 & No name & Asteraceae & Sonchus oleraceus L. & No use. \\
\hline 16243 & Hada & Asteraceae & Tagetes erecta $\mathrm{L}$. & Poison; kills cattle when they eat it. \\
\hline 16243 & Hada & Asteraceae & Tagetes erecta $\mathrm{L}$. & $\begin{array}{l}\text { Poison; very bad for humans. If it gets in a wound it will } \\
\text { expand. }\end{array}$ \\
\hline 16243 & Hada & Asteraceae & Tagetes erecta $\mathrm{L}$. & $\begin{array}{l}\text { Veterinary; used to treat cattle. Crush the leaves and put in the } \\
\text { hole a worm made to kill the worms (botfly remedy). }\end{array}$ \\
\hline 16320 & Sojom & Asteraceae & Vernonia amygdalina Delile & No use. \\
\hline 16338 & Ebicha & Asteraceae & Vernonia amygdalina Delile & Construction (timber). \\
\hline 16338 & Ebicha & Asteraceae & Vernonia amygdalina Delile & Eaten by cattle. \\
\hline 16338 & Ebicha & Asteraceae & Vernonia amygdalina Delile & Firewood. \\
\hline 16338 & Ebicha & Asteraceae & Vernonia amygdalina Delile & $\begin{array}{l}\text { Veterinary; smash leaves and feed to cattle for stomach } \\
\text { problems. }\end{array}$ \\
\hline 16021 & Regi & Asteraceae & Vernonia sp. & Flowers for honey. \\
\hline 16021 & Regi & Asteraceae & Vernonia sp. & $\begin{array}{l}\text { Veterinary; the leaves are fed to cattle who have stomach } \\
\text { problems to fatten them. }\end{array}$ \\
\hline 16032 & Gadarra & Asteraceae & Vernonia sp. & Eaten by cattle. \\
\hline 16053 & Kadara & Asteraceae & Vernonia sp. & Eaten by cattle and mountain nyala. \\
\hline 16053 & Kadara & Asteraceae & Vernonia sp. & Poison; the hairs can cause eye problems. \\
\hline 16212 & No name & Asteraceae & Vernonia sp. & No use. \\
\hline 16230 & Hevicha & Asteraceae & Vernonia sp. & Eaten by cattle. \\
\hline 16230 & Hevicha & Asteraceae & Vernonia sp. & $\begin{array}{l}\text { Veterinary; crush the leaves and make an extract. Give cattle to } \\
\text { drink when they are bloated. }\end{array}$ \\
\hline 16065 & Hadda & Asteraceae & & Eaten by cattle. \\
\hline 16091 & No name & Asteraceae & & No use. \\
\hline
\end{tabular}


Table 1 Plants encountered in Odo Bulu and Demaro, Bale, Ethiopia (Continued)

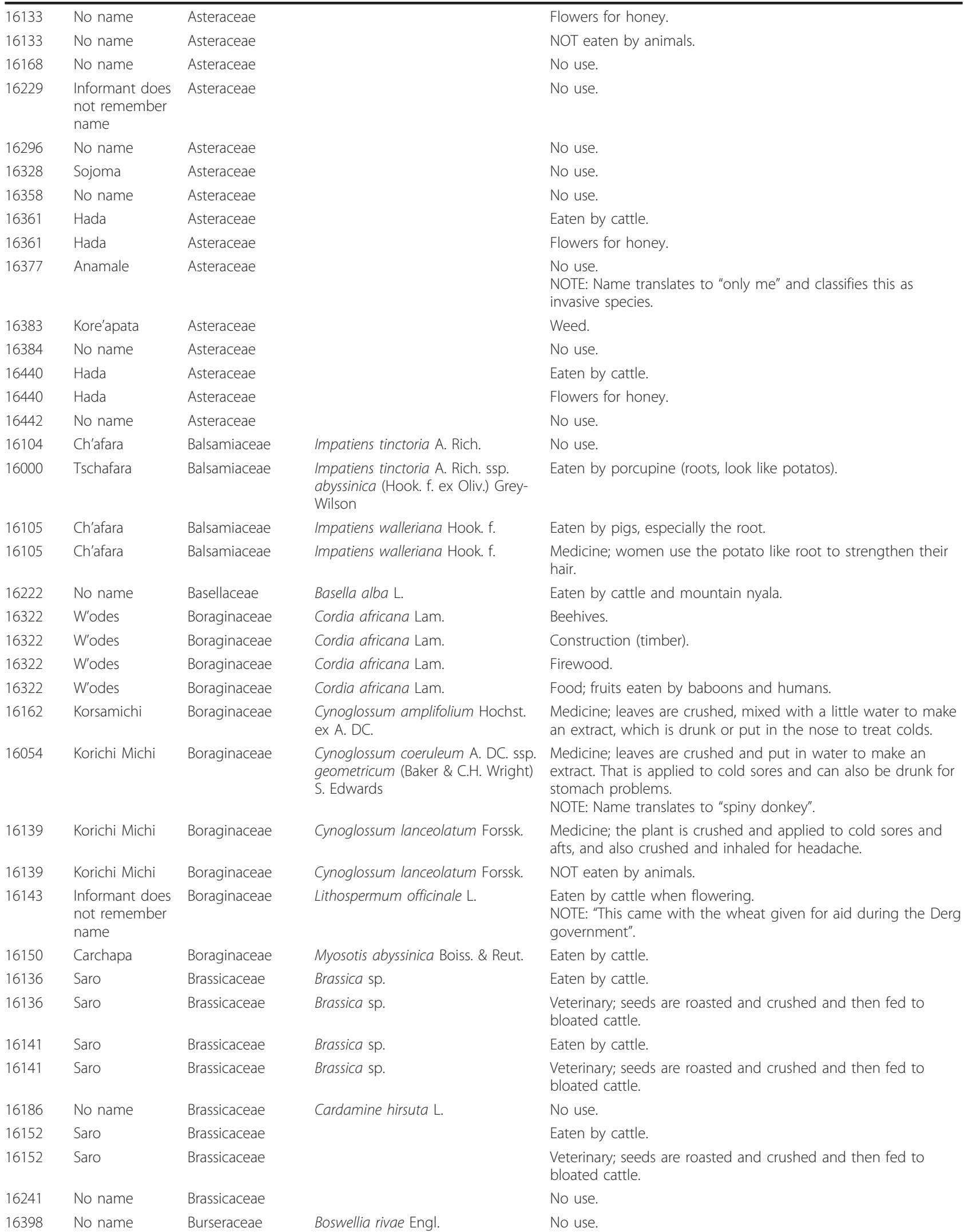


Table 1 Plants encountered in Odo Bulu and Demaro, Bale, Ethiopia (Continued)

\begin{tabular}{|c|c|c|c|c|}
\hline 16308 & Matakoma & Cabnnabaceae & Celtis africana Burm. f. & Firewood. \\
\hline 16308 & Matakoma & Cabnnabaceae & Celtis africana Burm. f. & Tools; wood used to make ploughs. \\
\hline 16388 & Bireliko & Cabnnabaceae & Celtis gomphophylla Baker & Firewood. \\
\hline 16419 & Matacoma & Cabnnabaceae & Celtis sp. & Firewood. \\
\hline 16061 & No name & Campanulaceae & Lobelia of erlangeriana Engl. & Eaten by baboons. \\
\hline 16073 & No name & Campanulaceae & $\begin{array}{l}\text { Wahlenbergia silenoides Hochst. ex } \\
\text { A. Rich }\end{array}$ & Eaten by cattle. \\
\hline 16441 & No name & Campanulaceae & Wahlenbergia sp. & No use. \\
\hline 16155 & $\begin{array}{l}\text { Informant does } \\
\text { not remember } \\
\text { name }\end{array}$ & Caryophyllaceae & Arenaria serpyllifolia $\mathrm{L}$. & Eaten by cattle. \\
\hline 16155 & $\begin{array}{l}\text { Informant does } \\
\text { not remember } \\
\text { name }\end{array}$ & Caryophyllaceae & Arenaria serpyllifolia L. & Medicine; for spiritual things. \\
\hline 16350 & Ch'oge & Caryophyllaceae & Cerastium hirsutum Crantz & $\begin{array}{l}\text { Eaten by cattle. } \\
\text { NOTE: Informants specifically mention small flower and fruit. }\end{array}$ \\
\hline 16110 & No name & Caryophyllaceae & Cerastium indicum Wight \& Arn. & No use. \\
\hline 16238 & No name & Caryophyllaceae & $\begin{array}{l}\text { Drymaria cordata (L.) Willd. ex } \\
\text { Roem. \& Schult. }\end{array}$ & Eaten by cattle. \\
\hline 16111 & Dukusha & Caryophyllaceae & Stellaria sennii Chiov. & $\begin{array}{l}\text { To soften leather. Crush the plant and apply to leather to make } \\
\text { leather ropes soft. }\end{array}$ \\
\hline 16077 & No name & Caryophyllaceae & & Eaten by baboons. \\
\hline 16026 & Kombocha & Celastraceae & Maytenus arbutifolia R. Wilczek & Eaten by livestock and mountain nyala. \\
\hline 16235 & Kombolcha & Celastraceae & Maytenus arbutifolia R. Wilczek & Eaten by cattle. \\
\hline 16235 & Kombolcha & Celastraceae & Maytenus arbutifolia R. Wilczek & Firewood. \\
\hline 16344 & Kombolcha & Celastraceae & Maytenus senegalensis (Lam.) Exell & Eaten by goats and mountain nyala \\
\hline 16344 & Kombolcha & Celastraceae & Maytenus senegalensis (Lam.) Exell & Firewood. \\
\hline 16344 & Kombolcha & Celastraceae & Maytenus senegalensis (Lam.) Exell & NOT eaten by cattle. \\
\hline 16195 & Kombolcha & Celastraceae & Maytenus sp. & Eaten by cattle and mountain nyala. \\
\hline 16195 & Kombolcha & Celastraceae & Maytenus sp. & Firewood. \\
\hline 16266 & Kombolcha & Celastraceae & Maytenus sp. & Eaten by goats. \\
\hline 16266 & Kombolcha & Celastraceae & Maytenus sp. & Flowers for honey. \\
\hline 16407 & Jima & Celastraceae & & Firewood. \\
\hline 16146 & Bucha & Chenopodiaceae & Chenopodium sp. & $\begin{array}{l}\text { Eaten by cattle, but if they eat it in the morning they get } \\
\text { bloated. }\end{array}$ \\
\hline 16148 & Bucha & Chenopodiaceae & Chenopodium sp. & $\begin{array}{l}\text { Eaten by cattle, but if they eat it in the morning they get } \\
\text { bloated. }\end{array}$ \\
\hline 16353 & No name & Chenopodiaceae & Chenopodium sp. & NOT eaten by animals. \\
\hline 16033 & Garramba & Clusiaceae & Hypericum revolutum Vahl & Construction; timber used for house posts. \\
\hline 16033 & Garramba & Clusiaceae & Hypericum revolutum Vahl & $\begin{array}{l}\text { Medicine; leaves boiled and given to babies with stomach } \\
\text { problems and to make babies stop crying. }\end{array}$ \\
\hline 16076 & Sedisa & Clusiaceae & Hypericum sp. & $\begin{array}{l}\text { Eaten by cattle. } \\
\text { NOTE: Informants were not completely sure about the name. }\end{array}$ \\
\hline 16404 & Tantefensa & Combretaceae & Combretum sp. & Charcoal. \\
\hline 16404 & Tantefensa & Combretaceae & Combretum sp. & Firewood. \\
\hline 16209 & No name & Commelinaceae & $\begin{array}{l}\text { Commelina imberbis Ehrenb. ex. } \\
\text { Hassk. }\end{array}$ & No use. \\
\hline 16366 & K'aio & Commelinaceae & Commelina sp. & Eaten by cattle and mountain nyala. \\
\hline 16393 & K'aio & Commelinaceae & Commelina sp. & Eaten by cattle. \\
\hline 16078 & No name & Commelinaceae & $\begin{array}{l}\text { Cyanotis polyrrhiza Hochst. ex } \\
\text { Hassk. }\end{array}$ & Eaten by baboons. \\
\hline 16048 & Hananu & Convolvulaceae & Convolvulus kilimandschari Engl. & Eaten by cattle and especially fed to calves. \\
\hline 16239 & Anano & Convolvulaceae & Convolvulus sp. & Eaten by cattle and mountain nyala. \\
\hline 16349 & No name & Convolvulaceae & $\begin{array}{l}\text { Dichondra repens J.R. Forst. \& G. } \\
\text { Forst. }\end{array}$ & Construction; used to build fences because of the spines. \\
\hline 16311 & Anamo & Convolvulaceae & Ipomoea sp. & Eaten by cattle and mountain nyala. \\
\hline
\end{tabular}


Table 1 Plants encountered in Odo Bulu and Demaro, Bale, Ethiopia (Continued)

\begin{tabular}{|c|c|c|c|c|}
\hline 16410 & No name & Convolvulaceae & Ipomoea sp. & No use. \\
\hline 16173 & No name & Crassulaceae & Crassula alba Forssk. & Eaten by baboons (root). \\
\hline 16081 & No name & Crassulaceae & $\begin{array}{l}\text { Crassula of schimperi Fisch. \& A. } \\
\text { Mey. }\end{array}$ & $\begin{array}{l}\text { Eaten by baboons. } \\
\text { NOTE: "This grows on rocks." }\end{array}$ \\
\hline 16086 & An'chura & Crassulaceae & Kalanchoe petitiana A. Rich. & $\begin{array}{l}\text { Medicine; heat the leaves over the fire and apply to relax sore } \\
\text { muscles. }\end{array}$ \\
\hline 16086 & An'chura & Crassulaceae & Kalanchoe petitiana A. Rich. & $\begin{array}{l}\text { Veterinary; if cattle break their legs, warm the plant or leaves } \\
\text { over the fire and rub on the broken bone. }\end{array}$ \\
\hline 16068 & No name & Crassulaceae & Sedum baleensis M. Gilbert & No use. \\
\hline 16090 & Angudula & Crassulaceae & $\begin{array}{l}\text { Sedum cf churchillianum Robyns \& } \\
\text { Boutique }\end{array}$ & $\begin{array}{l}\text { Poison; in former times used to poison Hyenas. The plant } \\
\text { material is crushed and out in meat. This makes the Hyena } \\
\text { weak and intoxicated so that it can be killed. }\end{array}$ \\
\hline 15996 & No name & Crassulaceae & Umbilicus botryoides A. Rich. & $\begin{array}{l}\text { No use. } \\
\text { NOTE: "It grows on trees." }\end{array}$ \\
\hline 16444 & No name & Crassulaceae & & No use. \\
\hline 16359 & Han'chote & Cucurbitaceae & Cucumis ficifolius A. Rich. & $\begin{array}{l}\text { Medicine; the roots are crushed and drunk with coffee for } \\
\text { sudden illness, e.g. when someone colapses or has sudden } \\
\text { stomach problems. }\end{array}$ \\
\hline 16117 & Alola & Cucurbitaceae & Zehneria scabra (L.f.) Sond. & Eaten (fed especially to calves). \\
\hline 16117 & Alola & Cucurbitaceae & Zehneria scabra (L.f.) Sond. & To make ink. In former times crushed and mixed with charcoal. \\
\hline 16335 & Haloa & Cucurbitaceae & & Eaten by cattle. \\
\hline 16424 & Hindesa & Cupressaceae & Juniperus procera Hochst. ex Endl. & Construction. \\
\hline 16424 & Hindesa & Cupressaceae & Juniperus procera Hochst. ex Endl. & Firewood. \\
\hline 16250 & No name & Cuscutaceae & Cuscuta kilimanjari Oliv. & $\begin{array}{l}\text { Eaten by cattle and mountain nyala. } \\
\text { NOTE: Recognized as parasite. }\end{array}$ \\
\hline 16250 & No name & Cuscutaceae & Cuscuta kilimanjari Oliv. & $\begin{array}{l}\text { Flowers for honey. } \\
\text { NOTE: Recognized as parasite. }\end{array}$ \\
\hline 16219 & D'jafa & Cyperaceae & $\begin{array}{l}\text { Carex of simensis Hochst. ex A. } \\
\text { Rich. }\end{array}$ & Thatch. \\
\hline 16006 & Mata & Cyperaceae & Carex johnstonii Boeck. & Eaten by animals. \\
\hline 16006 & Mata & Cyperaceae & Carex johnstonii Boeck. & Thatch. \\
\hline 16084 & Ch'afa & Cyperaceae & $\begin{array}{l}\text { Cyperus of bracheilema (Steud.) } \\
\text { Mattf. \& Kük. }\end{array}$ & Eaten by cattle. \\
\hline 16218 & Arbagadda & Cyperaceae & $\begin{array}{l}\text { Cyperus dichroostachyus Hochst. ex } \\
\text { A. Rich. }\end{array}$ & Eaten by cattle. \\
\hline 16218 & Arbagadda & Cyperaceae & $\begin{array}{l}\text { Cyperus dichroostachyus Hochst. ex } \\
\text { A. Rich. }\end{array}$ & Weave floor mats. \\
\hline 16345 & Bidara & Cyperaceae & Cyperus sp. & Eaten by cattle. \\
\hline 16085 & No name & Cyperaceae & Isolepis of omissa J. raynal & Eaten by baboons. \\
\hline 16023 & $\begin{array}{l}\text { Gwemagni/ } \\
\text { Talandu }\end{array}$ & Cyperaceae & Isolepis setacea (L.) R. Br. & Eaten by cattle. \\
\hline 16023 & $\begin{array}{l}\text { Gwemagni/ } \\
\text { Talandu }\end{array}$ & Cyperaceae & Isolepis setacea (L.) R. Br. & Thatch. \\
\hline 16292 & Aneno & Dioscoreaceae & Dioscorea sp. & Eaten by cattle and mountain nyala. Cattle really like this. \\
\hline 16179 & No name & Dipsacaceae & $\begin{array}{l}\text { Dipsacus pinnatifidus Steud. ex A. } \\
\text { Rich. }\end{array}$ & No use. \\
\hline 16123 & No name & Dipsacaceae & Scabiosa columbaria L. & No use. \\
\hline 16024 & No name & Dracaenaceae & Dracaena afromontana Mildbr. & No use. \\
\hline 16412 & Kokosa & Dryopteridaceae & $\begin{array}{l}\text { Doryopteris concolor } \\
\text { (Langsd. \& Fisch.) Kuhn }\end{array}$ & No use. \\
\hline 16016 & Kokosa & Dryopteridaceae & Dryopteris sp. & Sometimes eaten by cattle and mountain nyala. \\
\hline 16100 & Kokosa & Dryopteridaceae & Dryopteris sp. & No use. \\
\hline 16199 & Kokosa & Dryopteridaceae & Dryopteris sp. & No use. \\
\hline
\end{tabular}


Table 1 Plants encountered in Odo Bulu and Demaro, Bale, Ethiopia (Continued)

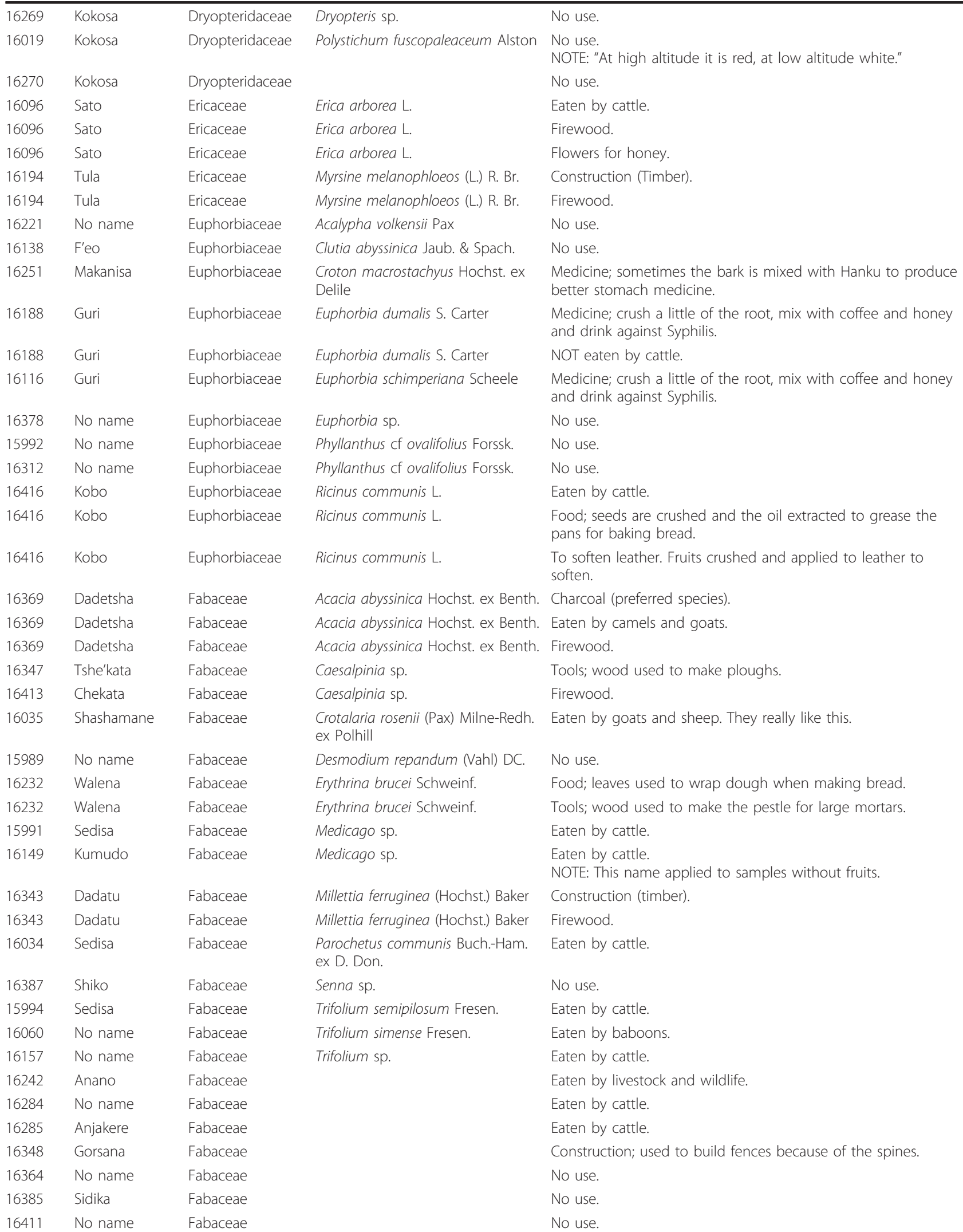


Table 1 Plants encountered in Odo Bulu and Demaro, Bale, Ethiopia (Continued)

\begin{tabular}{|c|c|c|c|c|}
\hline 16439 & Anjakere & Fabaceae & & Making brooms. \\
\hline 16406 & Dembi & Flacourtiaceae & Casearia sp. & Firewood. \\
\hline 16208 & $\begin{array}{l}\text { Koshimo/ } \\
\text { Koshima }\end{array}$ & Flacourtiaceae & Dovyalis abyssinica (A. Rich.) Warb. & Eaten by cattle. \\
\hline 16208 & $\begin{array}{l}\text { Koshimo/ } \\
\text { Koshima }\end{array}$ & Flacourtiaceae & Dovyalis abyssinica (A. Rich.) Warb. & Firewood. \\
\hline 16208 & $\begin{array}{l}\text { Koshimo/ } \\
\text { Koshima }\end{array}$ & Flacourtiaceae & Dovyalis abyssinica (A. Rich.) Warb. & Food; fruits eaten, but they are very sour. \\
\hline 16321 & Riga & Flacourtiaceae & & Toothbrush. \\
\hline 16346 & Diki & Flacourtiaceae & & Eaten by cattle and mountain nyala. \\
\hline 16346 & Diki & Flacourtiaceae & & Rope; used to hang beehives. \\
\hline 16399 & No name & Flacourtiaceae & & No use. \\
\hline 16074 & No name & Gentianaceae & Sebaea brachyphylla Griseb. & No use. \\
\hline 16062 & No name & Gentianaceae & Swertia sp. & Eaten by baboons. \\
\hline 16126 & No name & Gentianaceae & Swertia sp. & No use. \\
\hline 16170 & No name & Gentianaceae & Swertia sp. & No use. \\
\hline 16109 & No name & Geraniaceae & Geranium aculeolatum Oliv. & Eaten by cattle and mountain nyala. \\
\hline 16080 & No name & Geraniaceae & Geranium arabicum Forssk. & Eaten by cattle. \\
\hline 16373 & No name & Geraniaceae & Geranium arabicum Forssk. & No use. \\
\hline 16438 & No name & Iridaceae & & No use. \\
\hline 16102 & Ch'afa & Juncaceae & Luzula johnstonii Buchenau & Eaten by cattle when the plant is very young. \\
\hline 16102 & Ch'afa & Juncaceae & Luzula johnstonii Buchenau & Thatch. \\
\hline 16295 & Sukaiahareti & Lamiaceae & $\begin{array}{l}\text { Achyrospermum schimperi (Briq.) } \\
\text { Perkins }\end{array}$ & No use. \\
\hline 16160 & Tosin & Lamiaceae & $\begin{array}{l}\text { Becium cf obovatum } \\
\text { (E. Mey. ex Benth.) N.E. Br. }\end{array}$ & $\begin{array}{l}\text { Food; used to make tea, as spice for butter, and as spice for } \\
\text { baso (roasted and ground barley). }\end{array}$ \\
\hline 16310 & Burasisa & Lamiaceae & $\begin{array}{l}\text { Clerodendrum myricoides (Hochst.) } \\
\text { R. Br. ex Vatke }\end{array}$ & Eaten by cattle. \\
\hline 16137 & $\begin{array}{l}\text { Informant does } \\
\text { not remember } \\
\text { name }\end{array}$ & Lamiaceae & Leonotis nepetifolia (L.) R. Br. & Food; children like to suck the nectar from the flowers. \\
\hline 16137 & $\begin{array}{l}\text { Informant does } \\
\text { not remember } \\
\text { name }\end{array}$ & Lamiaceae & Leonotis nepetifolia (L.) R. Br. & Medicine; crush the plant and take as tea for stomach ailments. \\
\hline 16225 & Urgo & Lamiaceae & Leucas martinicensis (Jacq.) R. Br. & Eaten by cattle. \\
\hline 16316 & $\begin{array}{l}\text { Korichi Michi/ } \\
\text { Damakase }\end{array}$ & Lamiaceae & Ocimum sp. & $\begin{array}{l}\text { Medicine; for intestinal infections like cold or flu. Crush the } \\
\text { leaves, add a little water, inhale juice or smear on body or } \\
\text { drunk with coffee. }\end{array}$ \\
\hline 16332 & Urgohare & Lamiaceae & Ocimum sp. & No use. \\
\hline 16128 & Tunto & Lamiaceae & Otostegia tomentosa A. Rich. & Birds get nectar. \\
\hline 16128 & Tunto & Lamiaceae & Otostegia tomentosa A. Rich. & NOT eaten by cattle. \\
\hline 16079 & Burri & Lamiaceae & Plectranthus sp. & Eaten by cattle and baboons. \\
\hline 16087 & No name & Lamiaceae & Plectranthus sp. & No use. \\
\hline 16097 & Burri & Lamiaceae & Plectranthus sp. & Eaten by cattle. \\
\hline 16286 & Damakasi & Lamiaceae & Plectranthus sp. & Eaten by cattle. \\
\hline 16286 & Damakasi & Lamiaceae & Plectranthus sp. & $\begin{array}{l}\text { Medicine; leaves are crushed and the extract drunk for colds, or } \\
\text { leaves are crushed and stuck in the nose to treat colds. }\end{array}$ \\
\hline 16287 & Urgo & Lamiaceae & Plectranthus sp. & Eaten by cattle. \\
\hline 16287 & Urgo & Lamiaceae & Plectranthus sp. & Medicine; leaves crushed and put on wounds. \\
\hline 16356 & Ocota & Lamiaceae & Salvia merjamie Forssk. & Eaten by cattle. \\
\hline 16356 & Ocota & Lamiaceae & Salvia merjamie Forssk. & $\begin{array}{l}\text { Medicine; leaves crushed and smeared on the body to treat any } \\
\text { unknown or undiagnosed illness. }\end{array}$ \\
\hline 16001 & Ocota & Lamiaceae & Salvia nilotica Juss. ex Jacq. & Eaten by cattle and mountain nyala. \\
\hline 16098 & No name & Lamiaceae & Satureja sp. & No use. \\
\hline 16177 & No name & Lamiaceae & Satureja sp. & No use. \\
\hline
\end{tabular}


Table 1 Plants encountered in Odo Bulu and Demaro, Bale, Ethiopia (Continued)

\begin{tabular}{|c|c|c|c|c|}
\hline 15997 & $\begin{array}{l}\text { Informant does } \\
\text { not remember } \\
\text { name }\end{array}$ & Lamiaceae & Stachys aculeolata Hook. f. & Eaten by cattle and mountain nyala. \\
\hline 16178 & No name & Lamiaceae & Stachys sp. & No use. \\
\hline 16163 & No name & Lamiaceae & Thymus schimperi Ronniger & Eaten by cattle. \\
\hline 15995 & Burri & Lamiaceae & & Flowers for honey. \\
\hline 15995 & Burri & Lamiaceae & & NOT eaten by anything. \\
\hline 16389 & Abaye & Lauraceae & $\begin{array}{l}\text { Ocotea kenyensis (Chiov.) Robyns \& } \\
\text { R. Wilczek }\end{array}$ & Firewood. \\
\hline 16391 & Apeyu & Lauraceae & & Construction (house). \\
\hline 16391 & Apeyu & Lauraceae & & Firewood. \\
\hline 16066 & No name & Liliaceae & & $\begin{array}{l}\text { Eaten by baboons who really like it, especially the roots. Fruit } \\
\text { smells like garlic. }\end{array}$ \\
\hline 16215 & No name & Loranthaceae & $\begin{array}{l}\text { Englerina woodfordioides } \\
\text { (Schweinf.) Balle ex M.G. Gilbert }\end{array}$ & No use. \\
\hline 16187 & No name & Lycopodiaceae & $\begin{array}{l}\text { Huperzia dacrydioides (Baker) Pic. } \\
\text { Serm. }\end{array}$ & No use. \\
\hline 16268 & Danisa & Malvaceae & Dombeya kirkii Mast. & Eaten by cattle. \\
\hline 16268 & Danisa & Malvaceae & Dombeya kirkii Mast. & Flowers for honey. \\
\hline 16268 & Danisa & Malvaceae & Dombeya kirkii Mast. & Rope. Bark peeled for rope used in construction. \\
\hline 16323 & Danisa & Malvaceae & Dombeya sp. & Eaten by cattle. \\
\hline 16323 & Danisa & Malvaceae & Dombeya sp. & Firewood. \\
\hline 16323 & Danisa & Malvaceae & Dombeya sp. & Flowers for honey. \\
\hline 16058 & Danisa & Malvaceae & Dombeya torrida Bamps. & Eaten by cattle. \\
\hline 16118 & Danisa & Malvaceae & Dombeya torrida Bamps. & Flowers for honey. \\
\hline 16331 & Matakoma & Malvaceae & Grewia sp. & Firewood. \\
\hline 16267 & Ishini & Malvaceae & Hibiscus sp. & Eaten by cattle and mountain nyala. \\
\hline 16267 & Ishini & Malvaceae & Hibiscus sp. & Rope; bark peeled for rope used in construction. \\
\hline 16318 & No name & Malvaceae & Hibiscus sp. & No use. \\
\hline 16003 & Amoja/Amocha & Malvaceae & $\begin{array}{l}\text { Kosteletzkya adoensis } \\
\text { (Hochst. ex A. Rich.) Mast. }\end{array}$ & Eaten by cattle and mountain nyala. \\
\hline 16003 & Amoja/Amocha & Malvaceae & $\begin{array}{l}\text { Kosteletzkya adoensis } \\
\text { (Hochst. ex A. Rich.) Mast. }\end{array}$ & Toothbrush. \\
\hline 16130 & Lita & Malvaceae & Malva sp. & Eaten by cattle. \\
\hline 16130 & Lita & Malvaceae & Malva sp. & $\begin{array}{l}\text { Medicine; in the past used to stimulate hair growth. Plant } \\
\text { crushed and applied to the hair. }\end{array}$ \\
\hline 16317 & Ishini & Malvaceae & Pavonia sp. & Eaten by cattle and mountain nyala. \\
\hline 16317 & Ishini & Malvaceae & Pavonia sp. & Rope. Bark peeled and used as rope for construction. \\
\hline 16067 & Hamoja & Malvaceae & $\begin{array}{l}\text { Sparmannia ricinocarpa } \\
\text { (Eckl. \& Zeyh.) Kuntze }\end{array}$ & Eaten by Giant forest hog. \\
\hline 16224 & Amoja & Malvaceae & Triumfetta rhomboidea Jacq. & Eaten by mountain nyala, but NOT eaten by cattle. \\
\hline 16211 & No name & Malvaceae & & No use. \\
\hline 16245 & Ishini & Malvaceae & & Flowers for honey. \\
\hline 16245 & Ishini & Malvaceae & & Rope; bark peeled for rope used in construction. \\
\hline 16298 & Injin & Malvaceae & & Eaten by cattle and mountain nyala. \\
\hline 16298 & Injin & Malvaceae & & Rope. Bark peeled and used as rope for construction. \\
\hline 16324 & Anunu & Meliaceae & Ekebergia capensis Sparm. & Firewood. \\
\hline 16234 & Harambe & Meliaceae & $\begin{array}{l}\text { Lepidotrichilia volkensii (Gürke) J.-F. } \\
\text { Leroy }\end{array}$ & Firewood. \\
\hline 16234 & Harambe & Meliaceae & $\begin{array}{l}\text { Lepidotrichilia volkensii (Gürke) J.-F. } \\
\text { Leroy }\end{array}$ & Tools; wood to make ploughs. \\
\hline 16259 & Hacho & Meliaceae & $\begin{array}{l}\text { Lepidotrichilia volkensii (Gürke) J.F. } \\
\text { Leroy }\end{array}$ & Firewood. \\
\hline 16390 & Hacho & Meliaceae & Trichilia dregeana Sond. & Firewood. \\
\hline 16309 & Bulala & Meliaceae & Turraea holstii Gürke & No use. \\
\hline
\end{tabular}


Table 1 Plants encountered in Odo Bulu and Demaro, Bale, Ethiopia (Continued)

\begin{tabular}{|c|c|c|c|c|}
\hline 16029 & Arambye & Melianthaceae & Bersama abyssinica Fresen. & Tools; wood to make ploughs. \\
\hline 16342 & Oroka & Melianthaceae & Bersama abyssinica Fresen. & Firewood. \\
\hline 16183 & Kalala & Menispermaceae & $\begin{array}{l}\text { Stephania abyssinica } \\
\text { (Quart.-Dill. \& A. Rich.) Walp. }\end{array}$ & Rope; to tie houseposts. \\
\hline 16183 & Kalala & Menispermaceae & $\begin{array}{l}\text { Stephania abyssinica } \\
\text { (Quart.-Dill. \& A. Rich.) Walp. }\end{array}$ & Tools; to make containers for miliking. \\
\hline 16203 & No name & Moraceae & Dorstenia soerensenii Friis & No use. \\
\hline 16403 & Lint'o & Moraceae & Ficus sp. & Firewood. \\
\hline 16340 & Oda & Moraceae & Ficus sur Forssk. & Beehives (wood). \\
\hline 16340 & Oda & Moraceae & Ficus sur Forssk. & Food; fruits eaten by humans. \\
\hline 16340 & Oda & Moraceae & Ficus sur Forssk. & NOT eaten by cattle \\
\hline 16402 & Oda & Moraceae & Ficus sycomorus L. & Beehives (wood). \\
\hline 16402 & Oda & Moraceae & Ficus sycomorus L. & Food; fruits eaten by monkeys, baboons and humans. \\
\hline 16306 & No name & Moraceae & & No use. \\
\hline 16028 & Hanku & Myrsinaceae & Embelia schimperi Vatke & Eaten by goats, sheep, cattle, mountain nyala and baboons. \\
\hline 16028 & Hanku & Myrsinaceae & Embelia schimperi Vatke & $\begin{array}{l}\text { Medicine; the fruits and stems are used as anthelmintic. Eat } \\
\text { what fits in the bowl of a hand. }\end{array}$ \\
\hline 16260 & Hanku & Myrsinaceae & Embelia schimperi Vatke & Eaten by cattle and mountain nyala. \\
\hline 16260 & Hanku & Myrsinaceae & Embelia schimperi Vatke & Medicine; crush fruits and drink liquid to cure tapeworm. \\
\hline 16022 & Abeye & Myrsinaceae & Myrsine africana L. & $\begin{array}{l}\text { Food; women use the leaves to roll the dough in before } \\
\text { putting it in the oven so that it does not buirn. The seeds are } \\
\text { crused and the oil is used to grease the baking plate before } \\
\text { baking. }\end{array}$ \\
\hline 16272 & Badesa & Myrtaceae & Syzygium guineense (Willd.) DC. & Firewood. \\
\hline 16272 & Badesa & Myrtaceae & Syzygium guineense (Willd.) DC. & $\begin{array}{l}\text { Flowers for honey. } \\
\text { NOTE: Main honey source. }\end{array}$ \\
\hline 16272 & Badesa & Myrtaceae & Syzygium guineense (Willd.) DC. & Food; fruits eaten by humans. \\
\hline 16278 & Badesa & Myrtaceae & Syzygium guineense (Willd.) DC. & Food; fruits eaten by humans. \\
\hline 16405 & Badesa & Myrtaceae & Syzygium guineense (Willd.) DC. & Construction (house). \\
\hline 16405 & Badesa & Myrtaceae & Syzygium guineense (Willd.) DC. & Firewood. \\
\hline 16405 & Badesa & Myrtaceae & Syzygium guineense (Willd.) DC. & Food; fruit eaten by humans. \\
\hline 16374 & No name & Nyctaginaceae & Boerhavia sp. & No use. \\
\hline 16375 & No name & Nyctaginaceae & Boerhavia sp. & No use. \\
\hline 16112 & T'orso & Oleaceae & $\begin{array}{l}\text { Jasminum abyssinicum Hochst. ex. } \\
\text { DC. }\end{array}$ & Eaten (especially fed to calves). \\
\hline 16112 & T'orso & Oleaceae & $\begin{array}{l}\text { Jasminum abyssinicum Hochst. ex. } \\
\text { DC. }\end{array}$ & Eaten by cattle. \\
\hline 16112 & T'orso & Oleaceae & $\begin{array}{l}\text { Jasminum abyssinicum Hochst. ex. } \\
\text { DC. }\end{array}$ & Rope; used in house construction. \\
\hline 16112 & T'orso & Oleaceae & $\begin{array}{l}\text { Jasminum abyssinicum Hochst. ex. } \\
\text { DC. }\end{array}$ & Toothbrush (young stems). \\
\hline 16329 & Badesa & Oleaceae & Olea hochstetteri Baker & Construction (timber). \\
\hline 16329 & Badesa & Oleaceae & Olea hochstetteri Baker & Food; fruit eaten by humans. \\
\hline 16329 & Badesa & Oleaceae & Olea hochstetteri Baker & NOT eaten by cattle. \\
\hline 16124 & No name & Orobanchaceae & Orobanche minor Sm. & No use. \\
\hline 16106 & No name & Oxalidaceae & Oxalis sp. & Eaten by cattle. \\
\hline 16386 & Korehare & Papaveraceae & Argemone mexicana $\mathrm{L}$. & $\begin{array}{l}\text { Eaten by donkeys. } \\
\text { NOTE: Name translates to "spiny donkey". }\end{array}$ \\
\hline 16121 & Endode & Phytolaccaceae & Phytolacca dodecandra L.Hér. & Detergent; fruits crushed and used to wash cloths. \\
\hline 16121 & Endode & Phytolaccaceae & Phytolacca dodecandra L.Hér. & $\begin{array}{l}\text { Medicine; roots chewed for stomach problems. The fruits kill } \\
\text { water animals and can be used to prevent Bilharzia. }\end{array}$ \\
\hline 16121 & Endode & Phytolaccaceae & Phytolacca dodecandra L.Hér. & Toothbrush (twigs). \\
\hline
\end{tabular}


Table 1 Plants encountered in Odo Bulu and Demaro, Bale, Ethiopia (Continued)

\begin{tabular}{|c|c|c|c|c|}
\hline 16301 & No name & Phytolaccaceae & & Eaten by cattle. \\
\hline 16008 & Kontuyesa & Piperaceae & Peperomia abyssinica Miq. & $\begin{array}{l}\text { Medicine; boil the leaves and apply the steam to pimples and } \\
\text { abscesses. }\end{array}$ \\
\hline 16314 & No name & Piperaceae & $\begin{array}{l}\text { Peperomia tetraphylla (G. Forst.) } \\
\text { Hook. \& Arn. }\end{array}$ & No use. \\
\hline 16193 & Ara & Pittosporaceae & Pittosporum abyssinicum Delile & Eaten by cattle and mountain nyala. \\
\hline 16193 & Ara & Pittosporaceae & Pittosporum abyssinicum Delile & Firewood. \\
\hline 16193 & Ara & Pittosporaceae & Pittosporum abyssinicum Delile & Toothbrush (small branches). \\
\hline 16352 & $\begin{array}{l}\text { Name } \\
\text { unknown }\end{array}$ & Plantaginaceae & Plantago lanceolata L. & Eaten by cattle. \\
\hline 16428 & No name & Plantaginaceae & Plantago palmata Hook. f. & No use. \\
\hline 16005 & Garaba & Poaceae & Acritochaete volkensii Pilg. & Eaten by livestock and wildlife. \\
\hline 16434 & Tuta & Poaceae & Avenula sp. & Eaten by cattle. \\
\hline 16050 & Garaba & Poaceae & Bromus leptoclados Nees & Eaten by cattle. \\
\hline 16050 & Garaba & Poaceae & Bromus leptoclados Nees & Thatch. \\
\hline 16103 & Mata & Poaceae & Calamagrostis epigejos (L.) Roth. & NOT eaten by cattle. \\
\hline 16103 & Mata & Poaceae & Calamagrostis epigejos (L.) Roth. & Thatch. \\
\hline 16249 & Korcha & Poaceae & Digitaria sp. & Eaten by cattle and mountain nyala. \\
\hline 16253 & Hat'aua & Poaceae & Elymus sp. & Eaten by cattle. \\
\hline 16253 & Hat'aua & Poaceae & Elymus sp. & Thatch. \\
\hline 16082 & Marga & Poaceae & $\begin{array}{l}\text { Eragrostis cilianensis (Bellardi) } \\
\text { Vignolo ex. Janch. }\end{array}$ & $\begin{array}{l}\text { Eaten by cattle. } \\
\text { NOTE: Marga simply translates to grass. }\end{array}$ \\
\hline 16264 & Garaba & Poaceae & $\begin{array}{l}\text { Oplismenus compositus (L.) P. } \\
\text { Beauv. }\end{array}$ & Eaten by cattle and mountain nyala. \\
\hline 16299 & Marga & Poaceae & Panicum sp. & Eaten by cattle and mountain nyala. \\
\hline 16248 & Chokorsa & Poaceae & Paspalum sp. & Eaten by cattle and mountain nyala. \\
\hline 16290 & Babala/Babela & Poaceae & Paspalum sp. & Eaten by cattle and mountain nyala. \\
\hline 16351 & Marga & Poaceae & Poa annua L. & $\begin{array}{l}\text { Eaten by cattle. } \\
\text { NOTE: This plant had to be examined very closely before } \\
\text { consent could be reached. }\end{array}$ \\
\hline 16004 & Marga & Poaceae & Poa leptoclada A. Rich. & Eaten by cattle. \\
\hline 16262 & Babala/Babela & Poaceae & Stipa dregeana Steudl. & Eaten by cattle. \\
\hline 16049 & Garaba & Poaceae & $\begin{array}{l}\text { Streblochaete longiarista (A. Rich.) } \\
\text { Pilg. }\end{array}$ & Eaten by cattle. \\
\hline 16049 & Garaba & Poaceae & $\begin{array}{l}\text { Streblochaete longiarista (A. Rich.) } \\
\text { Pilg. }\end{array}$ & Thatch. \\
\hline 16265 & Garaba & Poaceae & $\begin{array}{l}\text { Streblochaete longiarista (A. Rich.) } \\
\text { Pilg. }\end{array}$ & Eaten by cattle and mountain nyala. \\
\hline 16083 & Marga & Poaceae & & $\begin{array}{l}\text { Eaten by cattle. } \\
\text { NOTE: Marga simply translates to grass. }\end{array}$ \\
\hline 16252 & Mata & Poaceae & & Eaten by cattle and mountain nyala. \\
\hline 16252 & Mata & Poaceae & & Thatch. \\
\hline 16291 & Babala/Babela & Poaceae & & Eaten by cattle and mountain nyala. \\
\hline 16300 & Marga & Poaceae & & Eaten by cattle and mountain nyala. \\
\hline 16433 & Mata & Poaceae & & Eaten by cattle. \\
\hline 16433 & Mata & Poaceae & & Thatch. \\
\hline 16246 & Bosoka & Polygonaceae & $\begin{array}{l}\text { Polygonum afromontanum } \\
\text { Greenway }\end{array}$ & No use. \\
\hline 16227 & Berberisa & Polygonaceae & Rumex abyssinicus Jacq. & No use. \\
\hline 16009 & Shabee & Polygonaceae & Rumex nepalensis Spreng. & Eaten by cattle. \\
\hline 16009 & Shabee & Polygonaceae & Rumex nepalensis Spreng. & Medicine; roots are crushed and eaten for stomach problems. \\
\hline 16009 & Shabee & Polygonaceae & Rumex nepalensis Spreng. & $\begin{array}{l}\text { Veterinary; leaves are crushed and given to livestock for } \\
\text { stomach problems. }\end{array}$ \\
\hline 16360 & Haberira & Polygonaceae & Rumex sp. & $\begin{array}{l}\text { Medicine; root is crushed, and water added and drunk when } \\
\text { somebody "feels that something is worng," i.e. has an } \\
\text { undiagnosed illness. }\end{array}$ \\
\hline
\end{tabular}


Table 1 Plants encountered in Odo Bulu and Demaro, Bale, Ethiopia (Continued)

\begin{tabular}{|c|c|c|c|c|}
\hline 16189 & Kokosa & Polypodiaceae & Drynaria volkensii Hieron. & No use. \\
\hline 16018 & No name & Polypodiaceae & $\begin{array}{l}\text { Loxogramme abyssinica (Baker) M. } \\
\text { G. Price }\end{array}$ & $\begin{array}{l}\text { No use. } \\
\text { NOTE: "At high altitude it is red, at low altitude white." }\end{array}$ \\
\hline 16392 & No name & Portulacaceae & Portulaca oleracea L. & No use. \\
\hline 16070 & No name & Primulaceae & Ardisiandra wettsteinii R. Wagner & Eaten by mountain nyala. \\
\hline 16154 & Matane & Primulaceaea & Anagallis arvensis $\mathrm{L}$. & $\begin{array}{l}\text { Eaten by cattle. } \\
\text { NOTE: "There are two kinds in the area." }\end{array}$ \\
\hline 16154 & Matane & Primulaceaea & Anagallis arvensis $\mathrm{L}$. & $\begin{array}{l}\text { Medicine. Applied to the eye to improve "fat yellow thing" in } \\
\text { the eye. } \\
\text { NOTE: "There are two kinds in the area." }\end{array}$ \\
\hline 16119 & No name & Pteridaceae & Adiantum capillus-veneris $\mathrm{L}$. & No use. \\
\hline 16101 & Kokosa & Pteridaceae & Cheilanthes farinosa (Forssk.) Kaulf. & No use. \\
\hline 16200 & Kokosa & Pteridaceae & Cheilanthes farinosa (Forssk.) Kaulf. & No use. \\
\hline 16217 & No name & Pteridaceae & Pteridium aquilinum (L.) Kuhn & No use. \\
\hline 16015 & Kokosa & Pteridaceae & Pteris catoptera Kunze & Sometimes eaten by cattle and mountain nyala. \\
\hline 16271 & Kokosa & Pteridaceae & Pteris catoptera Kunze & No use. \\
\hline 16261 & Kokosa & Pteridaceae & Pteris dentata Forssk. & No use. \\
\hline 16201 & Kokosa & Pteridaceae & Pteris flabellata Thunb. & No use. \\
\hline 16420 & Kokosa & Pteridaceae & Pteris sp. & No use. \\
\hline 16396 & Kokosa & Pteridaceae & & No use. \\
\hline 16047 & Fidy & Ranunculaceae & Clematis bracteata (Roxb.) Kurz & $\begin{array}{l}\text { Medicine; used for "lung cancer" and cancer in general. The } \\
\text { plant is crushed and mixed with butter and applied to wounds, } \\
\text { haemorrhoids and burns. }\end{array}$ \\
\hline 16282 & Hacho & Ranunculaceae & Clematis hirsuta Guill. \& Perr. & Eaten by cattle and mountain nyala. \\
\hline 16304 & Fidy & Ranunculaceae & Clematis simensis Fresen. & Rope; bark peeled and used as rope for construction. \\
\hline 16059 & No name & Ranunculaceae & Delphinium wellbyi Hemsl. & No use. \\
\hline 16427 & No name & Resedaceae & $\begin{array}{l}\text { Caylusea abyssinica Fisch. \& C.A. } \\
\text { Mey. }\end{array}$ & No use. \\
\hline 16325 & No name & Rhamnaceae & Helinus integrifolius (Lam.) Kuntze & No use. \\
\hline 16431 & Gesho & Rhamnaceae & Rhamnus prinoides L'Hér. & $\begin{array}{l}\text { Food; leaves used to flavor beer and mead. Only used by } \\
\text { Christians. Very bitter. }\end{array}$ \\
\hline 16365 & Bitana & Rhamnaceae & Rhamnus staddo A. Rich. & Construction (house). \\
\hline 16045 & Thelo & Rhizophoraceae & $\begin{array}{l}\text { Cassipourea malosana (Baker) } \\
\text { Alston }\end{array}$ & Firewood. \\
\hline 16045 & Thelo & Rhizophoraceae & $\begin{array}{l}\text { Cassipourea malosana (Baker) } \\
\text { Alston }\end{array}$ & Tools; wood used to make ploughs. \\
\hline 16169 & Sedisa & Rosaceae & Alchemilla fischeri Engl. & Eaten by cattle and baboons. \\
\hline 16181 & Sedisa & Rosaceae & Alchemilla kiwuensis Engl. & Eaten by cattle. \\
\hline 16057 & Heto & Rosaceae & Hagenia abyssinica J.F. Gmel. & Eaten by cattle. \\
\hline 16057 & Heto & Rosaceae & Hagenia abyssinica J.F. Gmel. & $\begin{array}{l}\text { Medicine; fruits are dried, ground and eaten before a meal as } \\
\text { anthelmintic. Better taken early in the morning with some food. }\end{array}$ \\
\hline 16226 & Suke & Rosaceae & Prunus africana (Hook. f.) Kalkman & Rope; used to hang beehives. \\
\hline 16192 & Gora & Rosaceae & Rubus steudneri Schweinf. & Eaten especially by mountain nyala. \\
\hline 16192 & Gora & Rosaceae & Rubus steudneri Schweinf. & Food; Fruit eaten by people. \\
\hline 16030 & Coralla & Rubiaceae & Canthium oligocarpum Hiern & Eaten by cattle and mountain nyala. \\
\hline 16030 & Coralla & Rubiaceae & Canthium oligocarpum Hiern & Food; fruits eaten by children. \\
\hline 16275 & Coralla & Rubiaceae & Canthium sp. & Firewood. \\
\hline 16275 & Coralla & Rubiaceae & Canthium sp. & Food; fruits eaten by humans. \\
\hline 16276 & Gagama & Rubiaceae & Canthium sp. & Tools; wood used to make ploughs. \\
\hline 16305 & Buna & Rubiaceae & Coffea arabica $\mathrm{L}$. & Food; fallen leaves roasted for tea, seeds coffee. \\
\hline 16180 & Matane & Rubiaceae & Galium aparinoides Forssk. & Eaten by cattle. \\
\hline 16164 & No name & Rubiaceae & $\begin{array}{l}\text { Oldenlandia monanthos } \\
\text { (Hochst. ex A. Rich.) Hiern }\end{array}$ & Eaten by cattle. \\
\hline 16051 & No name & Rubiaceae & $\begin{array}{l}\text { Pentas schimperiana (A. Rich.) } \\
\text { Vatke }\end{array}$ & Eaten by cattle. \\
\hline
\end{tabular}


Table 1 Plants encountered in Odo Bulu and Demaro, Bale, Ethiopia (Continued)

\begin{tabular}{|c|c|c|c|c|}
\hline 16283 & No name & Rubiaceae & Pentas sp. & Eaten by cattle and other animals. \\
\hline 16415 & Bulala & Rubiaceae & Psydrax sp. & Firewood. \\
\hline 16258 & Farangasa & Rubiaceae & Rhytigynia sp. & Firewood. \\
\hline 16426 & No name & Rubiaceae & Rubia cordifolia L. & $\begin{array}{l}\text { Medicine for "snake spit that causes wounds." Root and leaves } \\
\text { are chewed and then spit on the affected area. }\end{array}$ \\
\hline 16255 & Sugurgura & Rubiaceae & Vangueria sp. & Construction; to link the main poles and the roof beams. \\
\hline 16307 & Arbo & Rutaceae & Citrus aurantium $\mathrm{L}$. & Food; fruits eaten by baboons and humans. \\
\hline 16233 & H'adesa & Rutaceae & Teclea nobilis Delile & NOT used for firewood. \\
\hline 16233 & H'adesa & Rutaceae & Teclea nobilis Delile & Tools; wood to make ploughs. \\
\hline 16257 & Adesa & Rutaceae & Teclea sp. & Firewood. \\
\hline 16274 & Adesa & Rutaceae & Teclea sp. & Firewood. \\
\hline 16315 & Harira & Rutaceae & Teclea sp. & Firewood. \\
\hline 16417 & Sadiqua & Rutaceae & Toddalia asiatica (L.) Lam. & No use. \\
\hline 16273 & Harera & Rutaceae & Vepris dainellii (Pic. Seerm.) Miziray & Firewood. \\
\hline 16401 & Gagama & Rutaceae & & Tools; wood used to make ploughs. \\
\hline 16436 & Bitana & Rutaceae & & Firewood. \\
\hline 16339 & Deerto & Santalaceae & Viscum triflorum DC. & No use. \\
\hline 16044 & Habarra & Sapindaceae & $\begin{array}{l}\text { Allophylus abyssinicus (Hochst.) } \\
\text { Radkl. }\end{array}$ & Food; Fruits eaten by children. \\
\hline 16044 & Habarra & Sapindaceae & $\begin{array}{l}\text { Allophylus abyssinicus (Hochst.) } \\
\text { Radkl. }\end{array}$ & $\begin{array}{l}\text { Medicine; Flowers for Honey; This honey has medicinal } \\
\text { properties and is good for stomach problems. }\end{array}$ \\
\hline 16277 & Aberra & Sapindaceae & $\begin{array}{l}\text { Allophylus abyssinicus (Hochst.) } \\
\text { Radkl. }\end{array}$ & Eaten by cattle. \\
\hline 16277 & Aberra & Sapindaceae & $\begin{array}{l}\text { Allophylus abyssinicus (Hochst.) } \\
\text { Radkl. }\end{array}$ & Food; Fruits eaten by humans and baboons. \\
\hline 16256 & Guduba & Sapotaceae & $\begin{array}{l}\text { Aningeria adolfi-friederici (Engl.) } \\
\text { Robyns \& G.C.C. Gillbert }\end{array}$ & Firewood. \\
\hline 16072 & No name & Saxifragaceae & Saxifraga sp. & Eaten by baboons. \\
\hline 16075 & No name & Saxifragaceae & Saxifraga sp. & No use. \\
\hline 16167 & No name & Scrophulariaceae & Bartsia petitiana (A. Rich.) Hemsl. & No use. \\
\hline 16437 & No name & Scrophulariaceae & Bartsia sp. & No use. \\
\hline 16092 & Bulchana & Scrophulariaceae & Buddleja polystachya Fresen. & Firewood. \\
\hline 16158 & Kankarasho & Scrophulariaceae & Craterostigma pumilum Hochst. & Eaten by baboons (root). \\
\hline 16158 & Kankarasho & Scrophulariaceae & Craterostigma pumilum Hochst. & Food; Roots chewed by humans for taste (like a sweet carrot) \\
\hline 16071 & No name & Scrophulariaceae & Craterostigma sp. & Eaten by baboons. \\
\hline 16156 & Bilike & Scrophulariaceae & $\begin{array}{l}\text { Cycniopsis humilis A. Bacjklund, } \\
\text { Asfaw \& Långström }\end{array}$ & Eaten by baboons (root). \\
\hline 16159 & No name & Scrophulariaceae & Hebenstretia angolensis Rolfe & No use. \\
\hline 16064 & No name & Scrophulariaceae & Rhabdotosperma sp. & No use. \\
\hline 16429 & Ashishira & Scrophulariaceae & Verbascum sinaiticum Benth. & Making brooms. \\
\hline 16069 & No name & Scrophulariaceae & Veronica abyssinica Fresen. & No use. \\
\hline 16107 & Da'ta & Scrophulariaceae & $\begin{array}{l}\text { Veronica glandulosa Hochst. ex } \\
\text { Benth. }\end{array}$ & Eaten by cattle. \\
\hline 16443 & Hada & Scrophulariaceae & & Eaten by cattle. \\
\hline 16443 & Hada & Scrophulariaceae & & Flowers for honey. \\
\hline 16445 & No name & Scrophulariaceae & & No use. \\
\hline 16140 & Ironto & Simaroubaceae & Brucea antidysenterica J.F. Mill. & $\begin{array}{l}\text { NOT eaten by animals, not even by ants. The smoke and the } \\
\text { plant smell bad. Can be used as insect repellent. }\end{array}$ \\
\hline 16363 & Banji & Solanaceae & Datura stramonium L. & $\begin{array}{l}\text { Medicine; Leaves are crushed and applied to abscesses with } \\
\text { pus, deep thorns, infected swollen wounds etc. This will extract } \\
\text { the thorn/pus. } \\
\text { NOTE: The name translates to "plant from the east" in Amharic: } \\
\text { Atafaris. It is also used as generic name for "drug". }\end{array}$ \\
\hline
\end{tabular}


Table 1 Plants encountered in Odo Bulu and Demaro, Bale, Ethiopia (Continued)

\begin{tabular}{|c|c|c|c|c|}
\hline 16363 & Banji & Solanaceae & Datura stramonium L. & $\begin{array}{l}\text { Poison. Seeds might acidentially get mixed with barley. If that is } \\
\text { eaten one gets intoxicated, the mouth gets dry, and the mind } \\
\text { gets confused. The effect starts as soon as the digestion has } \\
\text { happened. }\end{array}$ \\
\hline 16042 & Marraro & Solanaceae & Discopodium penninervium Hochst. & Eaten by horses. \\
\hline 16381 & Benjisaria & Solanaceae & Physalis sp. & Weed. \\
\hline 16319 & Hiddi & Solanaceae & Solanum incanum L. & Eaten by cattle and goats. \\
\hline 16319 & Hiddi & Solanaceae & Solanum incanum L. & Medicine; roots chewed for stomach health and sudden pain. \\
\hline 16319 & Hiddi & Solanaceae & Solanum incanum L. & Poison; fruits are toxic. \\
\hline 16370 & Hiddi & Solanaceae & Solanum incanum L. & Eaten by cattle (leaves). \\
\hline 16370 & Hiddi & Solanaceae & Solanum incanum L. & $\begin{array}{l}\text { Medicine; root is eaten for stomach problems of sudden onset. } \\
\text { Very bitter. }\end{array}$ \\
\hline 16231 & Hiddi & Solanaceae & Solanum macracanthum A. Rich. & Medicine; root chewed for stomach problems. \\
\hline 16231 & Hiddi & Solanaceae & Solanum macracanthum A. Rich. & NOT eaten by cattle. \\
\hline 16362 & Mijilo & Solanaceae & Solanum nigrum L. & Eaten by cattle. \\
\hline 16362 & Mijilo & Solanaceae & Solanum nigrum L. & Food; children eat the fruits. \\
\hline 16371 & $\begin{array}{l}\text { Mishilo } \\
\text { Huarabesa }\end{array}$ & Solanaceae & Solanum sp. & $\begin{array}{l}\text { No use. } \\
\text { NOTE: Name translates as "Hyena tomato". }\end{array}$ \\
\hline 16372 & Hiti'arbo & Solanaceae & Solanum sp. & $\begin{array}{l}\text { Detergent; fruits were formerly boiled and used as detergent to } \\
\text { wash cloths. }\end{array}$ \\
\hline 16129 & Unso & Solanaceae & Withania somnifera (L.) Dunal & $\begin{array}{l}\text { Medicine; roots are smashed or chewed like a carrot to treat } \\
\text { stomach problems. The leaves are burnt as incense as spiritual } \\
\text { medicine. }\end{array}$ \\
\hline 16129 & Unso & Solanaceae & Withania somnifera (L.) Dunal & NOT eaten by cattle. \\
\hline 16380 & Hunso & Solanaceae & & $\begin{array}{l}\text { Veterinary. When the yoke hurts the back of the oxen and } \\
\text { produces a swelling, crush the leaves and put on the swelling. }\end{array}$ \\
\hline 16118 & Danisa & Sterculiaceae & Dombeya torrida Bamps. & $\begin{array}{l}\text { Rope; bark peeled and used in construction. "They pull the } \\
\text { water transport system for rope". }\end{array}$ \\
\hline 16367 & Bitana & Stilbaceae & Nuxia congesta R. Br. ex Fresen. & Construction (house). \\
\hline 16367 & Bitana & Stilbaceae & Nuxia congesta R. Br. ex Fresen. & Firewood. \\
\hline 16367 & Bitana & Stilbaceae & Nuxia congesta R. Br. ex Fresen. & Flowers for honey. \\
\hline 16302 & Kokosa & Tectariaceae & Tectaria gemmifera (Fée) Alston & No use. \\
\hline 16394 & Kokosa & Thelypteridaceae & Thelypteris sp. & No use. \\
\hline 16395 & Kokosa & Thelypteridaceae & Thelypteris sp. & No use. \\
\hline 16432 & Didisa & Thymeleaceae & Gnidia glauca (Fresen.) Gilg & Firewood. \\
\hline 16432 & Didisa & Thymeleaceae & Gnidia glauca (Fresen.) Gilg & Rope; bark used to make rope. \\
\hline 16184 & No name & Urticaceae & Pilea johnstonii Oliv. & No use. \\
\hline 16185 & No name & Urticaceae & Pilea rivularis Wedd. & Eaten by baboons. \\
\hline 16240 & No name & Urticaceae & Pilea sp. & Eaten by cattle. \\
\hline 16056 & Halila & Urticaceae & Urera hypselodendron Wedd. & Eaten by cattle and wildlife. \\
\hline 16254 & Halila & Urticaceae & Urera hypselodendron Wedd. & No use. \\
\hline 16334 & Lalesa & Urticaceae & & No use. \\
\hline 16281 & Sukai & Verbenaceae & Lantana sp. & Eaten by cattle. \\
\hline 16281 & Sukai & Verbenaceae & Lantana sp. & Food; spice for butter, milk etc. \\
\hline 16425 & No name & Verbenaceae & Verbena sp. & No use. \\
\hline 16280 & No name & Verbenaceae & & No use. \\
\hline 16327 & Ulaga & Verbenaceae & & Firewood. \\
\hline 16327 & Ulaga & Verbenaceae & & Tools; wood used to make ploughs. \\
\hline 16435 & Dukunsha & Violaceae & Viola abyssinica Steud. ex Oliv. & $\begin{array}{l}\text { To soften leather. Leaves are crushed to extract the juice, which } \\
\text { is applied to hard leather. }\end{array}$ \\
\hline 16020 & Lelecha & Vitaceae & Cyphostemma sp. & $\begin{array}{l}\text { Poison; this burns your bowels when you eat it. NOT eaten by } \\
\text { animals. }\end{array}$ \\
\hline 16418 & No name & Vitaceae & Rhoicissus sp. & NOT eaten by cattle. \\
\hline 16063 & No name & Xanthorrhoeaceae & Asphodelus fistulosus L. & Eaten by baboons. \\
\hline 16172 & Lela & Xanthorrhoeaceae & Kniphofia foliosa Hochst. & No use. \\
\hline
\end{tabular}


Table 1 Plants encountered in Odo Bulu and Demaro, Bale, Ethiopia (Continued)

\begin{tabular}{|c|c|c|c|c|}
\hline 16151 & Ch'amare & Zygophyllaceae & Tribulus terrestris L. & Eaten by all animals. \\
\hline 16151 & Ch'amare & Zygophyllaceae & Tribulus terrestris L. & Toothbrush (root). \\
\hline 16036 & Seriti & & & Eaten sometimes by goats. \\
\hline 16149 & Sedisa & & & $\begin{array}{l}\text { Eaten by cattle. } \\
\text { NOTE: This name applied to samples WITH fruits }\end{array}$ \\
\hline 16279 & Wayebosa & & & Eaten by cattle. \\
\hline 16279 & Wayebosa & & & Flowers for honey. \\
\hline 16421 & Diki & & & Eaten by cattle. \\
\hline 16421 & Diki & & & Rope. \\
\hline
\end{tabular}

human purposes, although acculturated societies are shown to retain a much higher plant usage in order to treat common "modern" diseases such as sexually transmitted disease, as well as veterinary problems that are either stigmatized, for which western treatment does not prove effective, or for which cheap treatment cannot be found. Western style health care services as provided by governments and NGOs, in particular in rural areas, seem to have contributed to a decline in traditional knowledge, in part because the local population simply regards western medicine as more effective and safer, or as one of our Oromo informants put it "Sick people go to the clinic or cultural practitioner who prepares medicine from plants. Nowadays people mostly go to the clinic. The head of household knows herbs and they might use these, but nowadays most people prefer to go to the clinic. Traditional herbalists are already very old. The tradition is normally passed from the father to the son. Formerly people came from far like from Addis, and there is still a woman healer who is famous for treatments for example for parasites. Western medicine is more scientific and thus more reliable. Traditional medicine is often very painful, and can cause harm. Sometimes people die of traditional medicine. For their animals people prefer to go to the animal hospital. Traditional remedies are only used for rabies."

The knowledge of the Oromo population in both the highlands of Bale and the lower areas south of the massif were comparable. However, some profound differences

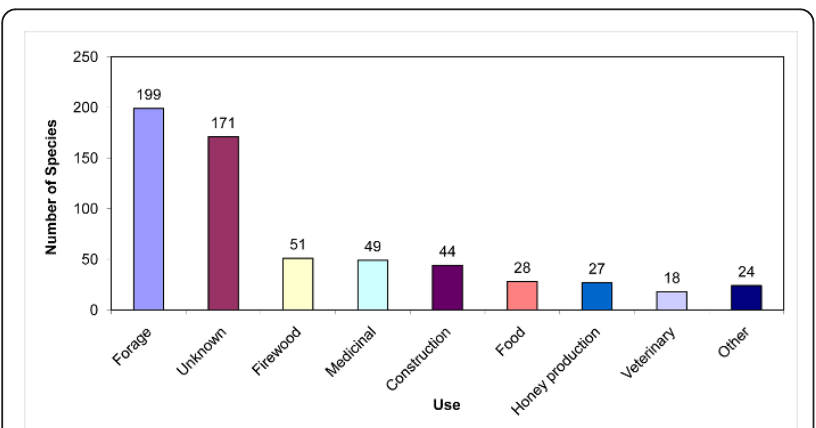

Figure 2 Number of plant species used in each use category. were encountered. The Oromo of the Bale highlands did not use preparations for malaria for the simple fact that malaria does not exist in their area. Thirteen species were used as veterinary medicine by the Oromo in the study area. This is rather surprising, because [42] reported 74 veterinary medicinal plant species from the study region. Plants for the cure of venereal diseases such as Gonorrhea, Syphilis and others, were almost negligible in the present study in the Oromo area. Previous records indicate that venereal diseases were amongst the most frequently treated with plants amongst the Oromo $[23,41]$.

These differences might indicate a clear difference in plant knowledge between traditional healers and laypeople. Experts clearly had a much more profound knowledge than the non-experts interviewed. We must also consider disparities in floral composition and availability between the Oromo people inhabiting our study area and those that inhabit different regions and ecosystems. In the worst case scenario, the Oromo in Bale may have already lost much of the plant knowledge that previous generations relied on for centuries.

\section{Acknowledgements}

The authors would like to thank all their colleagues in Ethiopia for their tireless support. We would like to thank in particular Sebsebe Demissew and Ensermu Kelbessa at the National Herbarium of Ethiopia for facilities to deposit specimens, help with identification, and literature. The financial support for this work by the Murulle Foundation, and the William L. Brown Center at Missouri Botanical Garden is acknowledged. Most of all, thanks to the population of Bale for sharing their tremendous ethnobotanical knowledge.

\section{Author details}

${ }^{1}$ William L. Brown Center, Missouri Botanical Garden, P.O. Box 299, St. Louis, MO 63166-0299, USA. ${ }^{2}$ The Murulle Foundaion, PO Box 1442, Fort Collins, CO 80522, USA. ${ }^{3}$ Rift Valley Safaris, PO Box 3658 Addis Ababa, Ethiopia. ${ }^{4}$ Natural Resource Ecology Laboratory, Colorado State University, Fort Collins, CO 80523-1499, USA.

\section{Authors' contributions}

$\mathrm{RB}$ and PS collected/identified plant material under the voucher acronym $\mathrm{RBU}$. RB, PS and AW conducted the interview work. RB analyzed the data and wrote the manuscript. PE elaborated on the Figures and the site description, and conducted the statistical analysis of the data as well as writing the manuscript. All authors have read and approved the final manuscript. 


\section{Competing interests}

The authors declare that they have no competing interests.

Received: 29 June 2011 Accepted: 24 September 2011

Published: 24 September 2011

\section{References}

1. Sindiga I, Nyaigotti-Chacha C, Kanunah MP: Traditional Medicine in Africa Nairobi, East African Educational Publishers; 1995.

2. Sindiga I, Kanunah MP, Aseka EM, Kiriga GW: Kikuyu traditional medicine. In Traditional Medicine in Kenya. Edited by: Sindiga I, Nyaigotti-Chacha C, Kanuna MP. Nairobi, East African Educational Publishers; 1995:

3. Barrow EGC: The dry lands of Africa: Local participation in tree management Nairobi, Initiative, Publishers; 1996.

4. Fratkin EM: Traditional medicine and concepts of healing among Samburu pastoralists of Kenya. Journal of Ethnobiology 1996, 16(1):63-97.

5. Abebe D, Ayehu A: Medicinal plants and enigmatic health practices of Northern Ethiopia Addis Ababa: B.S.P.E; 1993.

6. Abebe D: The role of medicinal plants in healthcare coverage of Ethiopia, the possible benefits of integration. In Conservation and sustainable use of medicinal plants in Ethiopia, Proceedings of the National workshop, 28 April-01 May 1998. Edited by: Zewdu M, Addis Ababa, Demissie A. Institute of Biodiversity Conservation and Research; 2001:6-21.

7. Abebe D: Traditional medicine in Ethiopia: the attempts being made to promote it for effective and better utilization. SINET 1986, 9:61-69.

8. Abebe G, Dawson L, Detweiler G, Gipson TA, Sahlu T: Hagenia abyssinica (Kosso) for internal parasite control in goats. In The opportunities and challenges of enhancing goat production in East Africa. Edited by: Merkel RC, Abebe G, Goetsch AL. E (Kika) de la Garza Institute for Goat research, Langston University, Langston, OK, Debub University, Awassa, Ethiopia; 2000:190-195.

9. Giday M, Asfaw Z, Elmqvist T, Woldu Z: An ethnobotanical study of medicinal plants used by the Zay People in Ethiopia. Journal of Ethnopharmacology 2003, 85:43-52.

10. Giday M, Teklehaymanot T, Animut A, Mekonnen Y: Medicinal plants of the Shinasha, Agew-awi and Amhara peoples in northwest Ethiopia. Journal of Ethnopharmacology 2007, 110:516-525.

11. Giday M, Asfaw Z, Woldu Z: Medicinal plants of the Meinit ethnic group of Ethiopia: An ethnobotanical survey. Journal of Ethnopharmacology 2009, 124:513-521.

12. Giday M, Asfaw Z, Woldu Z: An ethnobotanical study of plants used by the Sheko ethnic group of Ethiopia. Journal of Ethnopharmacology 2010, 132(1):75-85.

13. Teklehaymanot T, Giday M: Ethnobotanical study of medicinal plants used by people in Zegie Peninsula, northwestern Ethiopia. Journal of Ethnobiology and Ethnomedicine 2007, 3:12.

14. Teklehaymanot T: Ethnobotanical study of knowledge and medicinal plant use by people in Dek Island in Ethiopia. Journal of Ethnopharmacology 2009, 124:69-78.

15. Addis $G$, Abebe $D$, Genebo T, Urga K: Perceptions and practices of modern and traditional health practitioners about traditional medicine in Shirka District, Arsi Zone, Ethiopia. The Ethiopian Journal of Health Development 2002, 16:19-29.

16. Addis G, Abebe D, Urga K: A survey of traditional medicinal plants in Shirka District, Arsi Zone, Ethiopia. Ethiopian Pharmaceutical Journal 2001, 19:30-47.

17. Balemie K, Kebebew F: Ethnobotanical study of wild edible plants in Derashe and Kucha Districts, South Ethiopia. Journal of Ethnobiology and Ethnomedicine 2006, 5:23.

18. Balemie $K$, Kelbessa E, Asfaw Z: Indigenous medicinal plants utilization, management and threats in Fentale area, Eastern Shewa, Ethiopia. Ethiopian Journal of Biological Science 2004, 3:37-58.

19. Bekalo TH, Woodmatas SD, Woldemariam ZA: An ethnobotanical survey of medicinal plants used by local people in the lowlands of Konta Special Woreda, southern nations, nationalities and peoples regional state, Ethiopia. Journal of Ethnobiology and Ethnomecicine 2009, 5:26.

20. Flatie T, Gedig T, Asres K, Gebremariam T: Ethnomedicinal survey of Berta ethnic group, Assosa Zone, Benishangul-Gumuz regional state, midwestern Ethiopia. Journal of Ethnobiology and Ethnomedicine 2009, 5:14

21. Gedif T, Hahn H: The use of medicinal plants in self-care in rural central Ethiopia. Journal of Ethnopharmacology 2003, 87:155-161.
22. Geyid A, Abebe D, Debella A, Makonnen Z, Aberra F, Teka F, Kebede T, Urga K, Yersaw K, Biza T, Haile Mariam B, Gutta M: Screening of some medicinal plants of Ethiopia for their anti-microbial properties and chemical profiles. Journal of Ethnopharmacology 2005, 97:421-427.

23. Lulekal E, Kelbessa $\mathrm{E}$, Bekele T, Yineger $\mathrm{H}$ : An ethnobotanical study of medicinal plants in Mana Angetu District, southwestern Ethiopia. Journal of Ethnobiology and Ethnomedicine 2008, 4:10.

24. Teklehaymanot T, Giday M: Quantitative ethnobotany of medicinal plants used by the Kara and Kwego semi-pastoralist people in the Lower Omo River Valley, Debub Omo Zone, Southern Nations, Nationalities and Peoples Regional State, Ethiopia. Journal of Ethnopharmacology 2010, 130:76-84.

25. Yineger $\mathrm{H}$, Yewhalaw D: Traditional medicinal plant knowledge and use by local healers in Sekoru District, Jimma Zone, southwestern Ethiopia. Journal of Ethnobiology and Ethnomedicine 2007, 3:24.

26. Yonathan M, Asres K, Assefa A, Bucar F: In vivo anti-inflammatory and anti-nociceptive activities of Cheilanthes farinosa. Journal of Ethnopharmacology 2006, 108:462-470.

27. Demma J, Engidawork E, Hellman B: Potential genotoxicity of plant extracts used in Ethiopian traditional medicine. Journal of Ethnopharmacology 2009, 122:136-142.

28. Demma J, Gebremariam T, Asres K, Ergetie W, Engidawork E: Toxicology study on Glinus lotoides: A traditionally used taenicidal herb in Ethiopia. Journal of Ethnopharmacology 2007, 111:451-457.

29. Esser KB, Semagn K, Wode-Yohannes L: Medicinal use and social status of the soap berry endod (Phytolacca dodecandra) in Ethiopia. Journal of Ethnopharmacology 2003, 85:269-277.

30. Gebremariam T, Neubert R, Schmidt PC, Wutzler P, Schmidtke M: Antiviral activities of some Ethiopian medicinal plants used for the treatment of dermatological disorders. Journal of Ethnopharmacology 2006, 104(12):182-187.

31. Gebrie E, Makonnen E, Debella A, Zerihun L: Phytochemical screeing and pharmacological evaluations for the antifertility effect of the methanolic root extract of Rumex steudelii. Journal of Ethnopharmacology 2005, 96:139-143.

32. Makonnen E, Debella A, Zerihun L, Abebe D, Teka F: Antipyretic properties of the aqueous and ethanol extracts of the leaves of Ocimum suave and Ocimum lamiifiolium in mice. Journal of Ethnopharmacology 2003, 88:85-91.

33. Mazzanti G, Bolle P, Martinoli L, Piccinelli D, Grgurina I, Animati F, Mugnè Y: Croton macrostachys, a plant used in traditional medicine: purgative and inflammatory activity. Journal of Ethnopharmacology 1987, 19(2):213-219

34. Mekonnen T, Urga K, Engidawok E: Evaluation of the diuretic and analgesic activities of the rhizomes of Rumex abyssinicus Jacq. in mice. Journal of Ethnopharmacology 2010, 127:433-439.

35. Hillman JC: Bale Mountains National Park Management Plan. Ethiopian Wildlife Conservation Organization (EWCO), Addis Ababa 1986, 250.

36. Bussmann RW: The forest vegetation of the Harenna escarpment (Bale Province, Ethiopia) syntaxonomy and phytogeographical affinities. Phytocoenologia 1997, 27(1):1-23.

37. Miehe S, Miehe G: Ericaceous Forests and Heath Lands in the Bale Mountains of South Ethiopia: Ecology and Man's Impact Stiftung Walderhaltung, Hamburg; 1994, 206.

38. Williams S: Bale Mountains: A Guidebook EWCP, Addis Ababa, Ethiopia; 2002, 52.

39. Admassie Y: Twenty Years to Nowhere: Property Rights, Land Management and Conservation in Ethiopia Red Sea Press, Lawrenceville, NJ; 1998.

40. Karunamoorthi $\mathrm{K}$, llango K, Ebdale A: Ethnobotanical survey and usage custom of traditional insect/mosquito repellant plants among the Ethiopian Oromo ethic group. Journal of Ethnopharmacology 2009, 125:224-229.

41. Yineger $\mathrm{H}$, Yewhalaw D, Teketay D: Ethnomedicinal plant knowledge and practice of the Oromo ethnic group in souwthwestern Ethiopia. Journal of Ethnobiology and Ethnomedicine 2008, 4:11.

42. Yineger $\mathrm{H}$, Kelbessa $\mathrm{E}$, Bekele $\mathrm{T}$, Lulekal E: Ethnoveterinary medicinal plants at Bale Mountains National Park, Ethiopia. Journal of Ethnopharmacology 2007, 112(3):55-70.

43. Alexiades M: Collecting ethnobotanical data. An introduction to basic concepts and techniques. In Selected Guideline for Ethnobotanical Research: A Field Manual. Edited by: Alexiades M, Sheldon JW. The New York Botanical Garden, USA; 1996:53-94. 
44. Edwards S, Demissew S, Hedberg I, eds: Flora of Ethiopia and Eritrea. Hydrocharitaceae to Arecaceae Volume 6 The National Herbarium, Addis Ababa, Ethiopia, and Department of Systematic Botany, Uppsala, Sweden; 1997.

45. Edwards S, Tadesse M, Demissew S, Hedberg I, eds: Flora of Ethiopia and Eritrea. Magnoliaceae to Flacourtiaceae Volume 2 Issue part 1 The National Herbarium, Addis Ababa, Ethiopia, and Department of Systematic Botany, Uppsala, Sweden; 2000

46. Edwards S, Tadesse M, Hedberg I, eds: Flora of Ethiopia and Eritrea. Canellaceae to Euphorbiaceae Volume 2 Issue part 2 The National Herbarium, Addis Ababa, Ethiopia, and Department of Systematic Botany, Uppsala, Sweden; 1995.

47. Hedberg I, Edwards S, eds: Flora of Ethiopia and Eritrea. Pittosporaceae to Araliaceae Volume 3 The National Herbarium, Addis Ababa, Ethiopia, and Department of Systematic Botany, Uppsala, Sweden; 1989.

48. Hedberg I, Edwards S, eds: Flora of Ethiopia and Eritrea. Poaceae Volume 7 The National Herbarium, Addis Ababa, Ethiopia, and Department of Systematic Botany, Uppsala, Sweden; 1995.

49. Hedberg I, Edwards S, Nemomissa S, eds: Flora of Ethiopia and Eritrea. Apiaceae to Dipsacaceae Volume 4 Issue part 1 The National Herbarium, Addis Ababa, Ethiopia, and Department of Systematic Botany, Uppsala, Sweden; 2003

50. Hedberg I, Friii I, Edwards S, eds: Flora of Ethiopia and Eritrea. Asteraceae Volume 4 Issue part 2 The National Herbarium, Addis Ababa, Ethiopia, and Department of Systematic Botany, Uppsala, Sweden; 2004.

51. Azene BT, Brinie A, Tegnäs B: Useful trees and Shrubs for Ethiopia: Identification, Propagation and Management for Agricultural and Pastoral Communities Regional Conservation Unit, Swedish International Development Authority (SIDA); 1993.

52. Beentje HJ: Kenya trees, shrubs and lianas National Museums of Kenya, Nairobi; 1994.

53. Friis I: Forests and forest trees of Northeast Tropical Africa Kew Bulletin Additional Series XV, London, Kew Botanical Gardens: HMSO; 1992.

\section{doi:10.1186/1746-4269-7-28}

Cite this article as: Bussmann et al:: Plant use in Odo-Bulu and Demaro, Bale region, Ethiopia. Journal of Ethnobiology and Ethnomedicine 2011 7:28.

\section{Submit your next manuscript to BioMed Central and take full advantage of:}

- Convenient online submission

- Thorough peer review

- No space constraints or color figure charges

- Immediate publication on acceptance

- Inclusion in PubMed, CAS, Scopus and Google Scholar

- Research which is freely available for redistribution

Submit your manuscript at www.biomedcentral.com/submit 